\title{
Bioinspired Theranostic Coordination Polymer Nanoparticles for Intranasal Dopamine Replacement in Parkinson's Disease
}

\author{
Javier García-Pardo, Fernando Novio, Fabiana Nador, Ivana Cavaliere, Salvio Suárez-García, \\ Silvia Lope-Piedrafita, Ana Paula Candiota, Jordi Romero-Gimenez, Beatriz Rodríguez-Galván, \\ Jordi Bové, Miquel Vila, Julia Lorenzo,* and Daniel Ruiz-Molina*
}

ABSTRACT: Dopamine (DA) is one of the main neurotransmitters found in the central nervous system and has a vital role in the function of dopaminergic (DArgic) neurons. A progressive loss of this specific subset of cells is one of the hallmarks of age-related neurodegenerative disorders such as Parkinson's disease (PD). Symptomatic therapy for PD has been centered in the precursor LDOPA administration, an amino acid precursor of DA that crosses the blood-brain barrier (BBB) while DA does not, although this approach presents medium- to long-term side effects. To overcome this limitation, DA-nanoencapsulation therapies are actively being searched as an alternative for DA replacement. However, overcoming the low yield of encapsulation and/or poor biodistribution/ bioavailability of DA is still a current challenge. Herein, we report

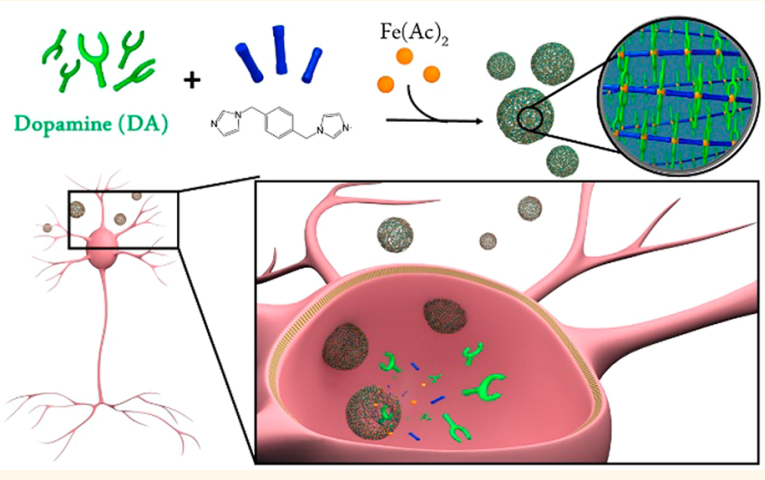
the synthesis of a family of neuromelanin bioinspired polymeric nanoparticles. Our system is based on the encapsulation of DA within nanoparticles through its reversible coordination complexation to iron metal nodes polymerized with a bis-imidazol ligand. Our methodology, in addition to being simple and inexpensive, results in DA loading efficiencies of up to $60 \%$. In vitro, DA nanoscale coordination polymers (DA-NCPs) exhibited lower toxicity, degradation kinetics, and enhanced uptake by BE(2)-M17 DArgic cells compared to free DA. Direct infusion of the particles in the ventricle of rats in vivo showed a rapid distribution within the brain of healthy rats, leading to an increase in striatal DA levels. More importantly, after 4 days of nasal administrations with DA-NCPs equivalent to $200 \mu \mathrm{g}$ of the free drug per day, the number and duration of apomorphine-induced rotations was significantly lower from that in either vehicle or DA-treated rats performed for comparison purposes. Overall, this study demonstrates the advantages of using nanostructured DA for DA-replacement therapy.

KEYWORDS: neuromelanin, dopamine, Parkinson's disease, neurodegeneration, coordination polymers

\section{INTRODUCTION}

Parkinson's disease (PD), the second most common neurodegenerative disorder in the world, affecting more than 10 million people, ${ }^{1,2}$ is characterized by motor symptoms including muscular rigidity, rest tremor, posture instability, and bradykinesia. ${ }^{1}$ The origin is associated with early prominent death of DArgic neurons typically of the substantia nigra pars compacta $(\mathrm{SNpc}),{ }^{3}$ which leads to diminished levels of dopamine (3,4-dihydroxyphenethylamine, DA) in the striatum of these patients. ${ }^{4-7}$ Mainly due to restrictions for direct DA replacement, delivery of the precursor levodopa ( $\mathrm{L}$ 3,4-dihydroxyphenylalanine, L-DOPA) has been used in the clinic for more than 50 years to mitigate Parkinson symptoms. ${ }^{1}$ However, long-term administration of this drug is associated with developing highly disabling complications such as drug-induced dyskinesia and motor fluctuations. ${ }^{8-10}$ Both complications are in part due to pulsatile L-DOPA administration and can be minimized with continuous and sustainable L-DOPA delivery by duodenal infusion. ${ }^{11}$ Evidence

Received: January 17, 2021

Accepted: April 12, 2021

Published: April 22, 2021

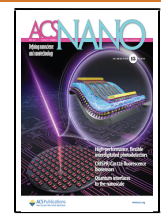


A

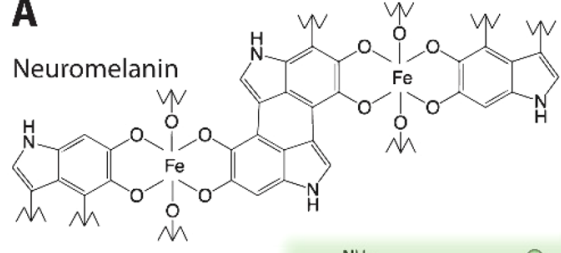

Our approach

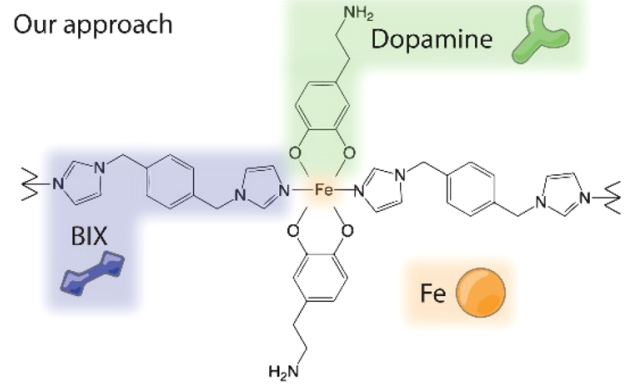

B

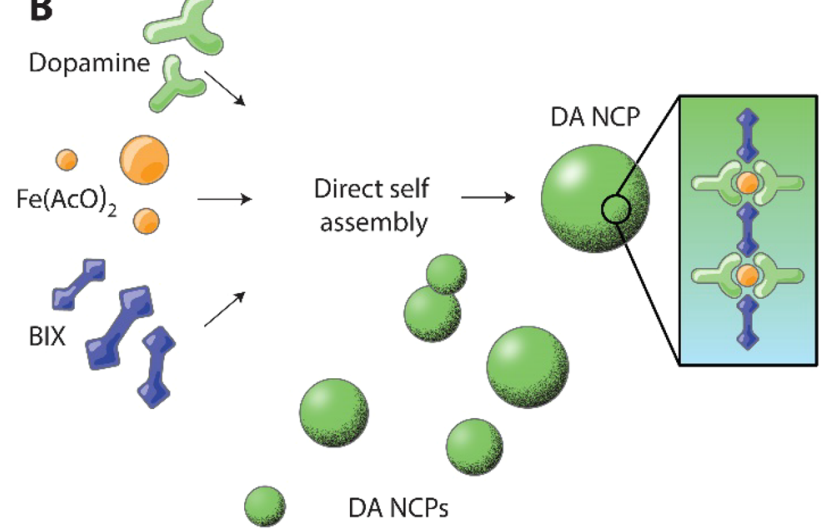

C
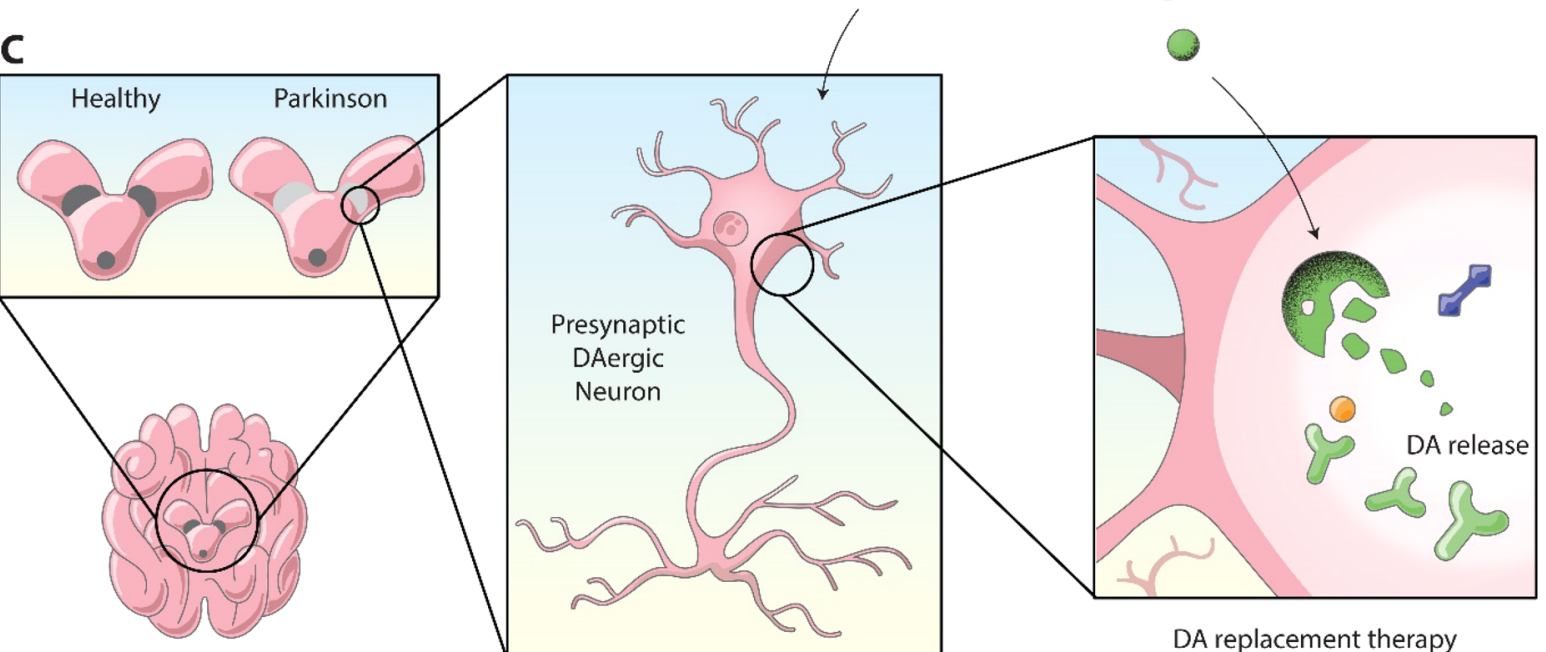

DA replacement therapy

Figure 1. Bioinspired approach for dopamine (DA) replacement in Parkinson's disease (PD). (a) Schematic composition of neuromelanin (NM) and the related nanoscale coordination polymer (DA-NCP) proposed herein. (b) Schematic representation of the synthesis of DANCPs from DA, Fe(AcO) $)_{2}$, and BIX. (c) Proposed model for DA-based replacement therapy in Parkinson's disease using DA-NCPs.

suggests that up to $50 \%$ of PD patients develop these complications after five years of chronic treatment with levodopa. Therefore, the development of alternative strategies to overcome these complications is an important challenge in modern medicine: among them, finding approaches for the delivery of DA through its nanoencapsulation.

Nanocarriers can help DA to avoid metabolism, cross the $\mathrm{BBB}$, and promote a sustained release, reducing recurrent drug administration and, therefore, adverse effects. So far, different families of nanocarriers have been described with this aim, including chitosan ${ }^{12}$ and transferrin ${ }^{13}$ functionalized liposomes, (bio)hydrogels, ${ }^{14,15}$ chitosan nanoparticles, ${ }^{16,17}$ carbon nanotubes, ${ }^{18}$ alginate/magnetite hybrid beads, ${ }^{19}$ carbon dots, ${ }^{20}$ carboxymethyl cellulose coated magnetic nanoparticles, ${ }^{21}$ borneol and lactoferrin co-modified nanoparticles, ${ }^{22}$ and a polyvinylpyrrolidone-poly(acrylic acid) nanogel. $^{23}$ The work by Gupta et al. deserves special mention, which reported DA-loaded poly lactic-co-glycolic acid (PLGA) nanoparticles able to reverse functional deficits in Parkinsonian rats. ${ }^{24}$ However, despite these advances, most of the nanocarriers used for DA replacement therapy often suffer from low yield of encapsulation and/or poor biodistribution and drug bioavailability, so alternative encapsulation approaches are required.
Neuromelanin (NM) particles, found in DArgic neurons from the SNpc, incorporate large amounts of DA and iron ions, which allow for their use as a biomarker for PD. Therefore, we hypothesize that melanin granular pigments found in nature may offer inspiration to develop more effective DA nanocarriers. ${ }^{25}$ To mimic the synthesis of melanin-like nanoparticles in the lab, whose tentative formula is shown in Figure $1 a^{26}$ three main approaches have been so far reported. In the first approach, self-oxidation of DA under alkaline conditions results in the formation of colloidal polyDA (PDA) nanoparticles, which subsequently are doped with the appropriate metal salt (postdoping approach). ${ }^{27-30}$ Alternatively, one-pot polymerization of DA in the presence of transition-metal ions results in their inclusion in the final PDA (in situ doping). ${ }^{31-33}$ And the third approach involves the preformation of $\mathrm{Fe}(\mathrm{DA})_{3}$ monomeric complexes that systematically control the percentage of iron in the particles. ${ }^{34-36}$ Following these approaches, synthetic nanoparticles showing excellent biocompatibility and biodegradability have already been prepared and successfully used in phototherapy/drug delivery $^{37}$ and imaging. ${ }^{38-40}$ However, none of them were found to be useful for the delivery of DA, as the polymerization and formation of the nanoparticles always take place through irreversible covalent bonds. 
a

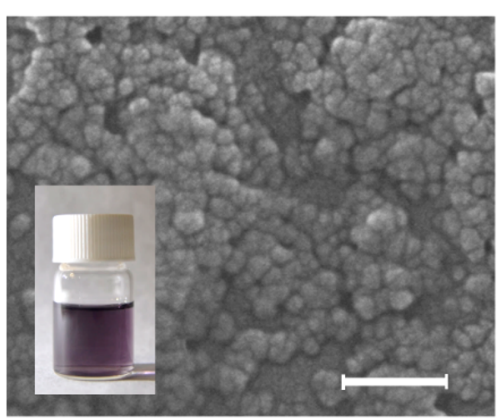

c

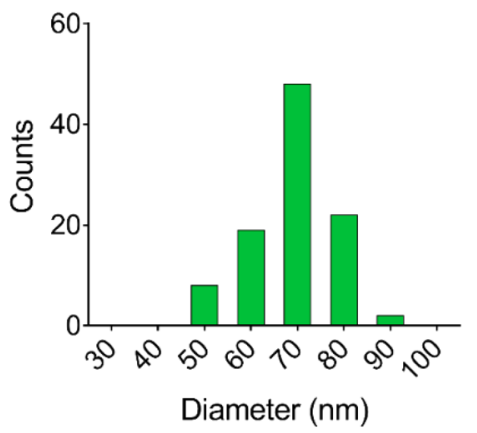

b

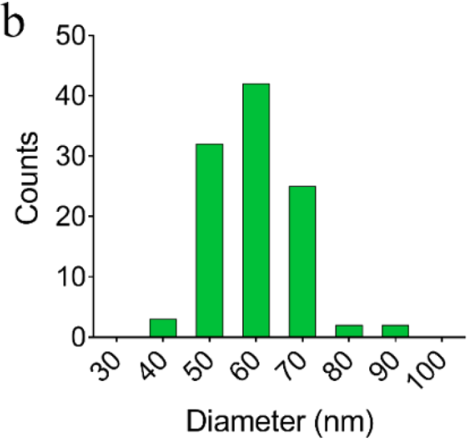

e

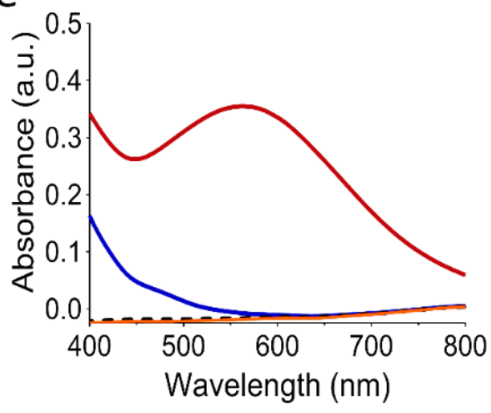

d
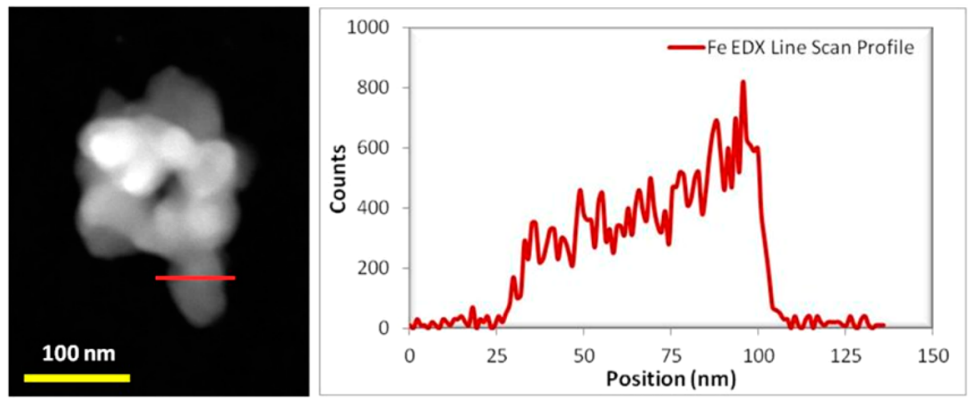

Figure 2. Chemical and morphological characterization of DA-NCPs. (a) SEM images of neuromelanin-inspired DA-NCPs. The inset shows a colloidal dispersion of the nanoparticles in ethanol. Scale bar, $200 \mathrm{~nm}$. (b, c) Particle size distribution of DA-NCPs determined by (b) SEM and (c) negative-staining transmission electron microscopy (NS-TEM). (d) TEM image (left) and EDX profile of DA-NCPs along the dark red line marked (right). (e) UV-vis absorbance spectra of the particles (solid dark red line) and the corresponding chemical precursors: DA (black dashed line), $\mathrm{BIX}$ (solid blue line), and $\mathrm{Fe}(\mathrm{AcO})_{2}$ (solid orange line) determined at a concentration of $100 \mu \mathrm{g} / \mathrm{mL}$ in water.

Herein we envision that this could be achieved with the formation of a tailor-made coordination polymer, where the central polymeric backbone is made of iron nodes linked to a bidentate ligand such as 1,4-bis(imidazol-1-ylmethyl)benzene (BIX) and DA is used as a counter ligand to complete the coordination sphere. The composition of the resulting nanoparticles (named from now on as DA-NCPs) is expected to have a structure inspired by NM, as shown in Figure 1a, but with the fundamental difference being that the incorporation of DA to the NP is reversible inside the cell. A schematic representation of the synthesis of the DA-NCPs nanoparticles and the expected biological function is shown in Figure 1b,c.

\section{RESULTS AND DISCUSSION}

Synthesis and Characterization of DA-NCPs. DANCPs were prepared following a procedure already described for related catechol-based nanoparticles. ${ }^{41,42}$ In a typical synthesis, nanoparticles were obtained through a reaction of $\mathrm{Fe}(\mathrm{AcO})_{2}$ with $\mathrm{DA}$ hydrochloride in the presence of the ditopic ligand BIX. After $2 \mathrm{~h}$ under constant stirring at room temperature, a dark precipitate was isolated by centrifugation and washed gently with ethanol to eliminate all the unbound ligands (see details in Methods and Supporting Information). Scanning electron microscopy (SEM) and negative-staining transmission electron microscopy (NS-TEM) images of the obtained material revealed the presence of spherical nanoparticles with an average diameter of $64 \pm 9.0 \mathrm{~nm}$ and $56 \pm$ $9.0 \mathrm{~nm}$, respectively (Figure 2a,c). These results were in agreement with dynamic light scattering (DLS) measurements of the DA-NCPs dispersed in ethanol, which showed a hydrodynamic diameter of $81 \pm 4.0 \mathrm{~nm}$ (PDI: 0.124) (Supporting Information, Figure S1). Energy-dispersive Xray analysis (EDX) profile of the TEM image clearly revealed the presence of iron homogeneously distributed along the whole nanoparticle (see Figure 2d), and Mössbauer spectroscopy studies confirmed that the iron was in the high-spin $\mathrm{Fe}$ (III) form (Supporting Information, Figure S2). This observation suggested that $\mathrm{Fe}$ (II) was oxidized to $\mathrm{Fe}(\mathrm{III})$ along with the nanoparticle synthesis. In agreement, the 
absorbance spectrum of DA-NCPs in the 400-800 nm range exhibited a characteristic maximum at $562 \mathrm{~nm}$, which was attributed to the formation of Fe-bis-catechol coordination bonds (Figure 2e). This electronic modification has been attributed to a redox interplay between the metal ion and electroactive catechol ligands in air, as previously reported. ${ }^{43}$ The absence of diffraction peaks observed by powder X-ray diffraction (PXRD) indicated the amorphous nature of the nanoparticles (see Supporting Information, Figure S3).

The chemical characterization of DA-NCPs was addressed using different complementary techniques. Fourier transform infrared spectroscopy (FT-IR) confirmed the presence of DA and a BIX ligand coordinated to the $\mathrm{Fe}$ (III) metal ions (see Supporting Information, Figure S4). The FT-IR spectrum shows the disappearance of the bands corresponding to the catechol $-\mathrm{OH}$ groups of $\mathrm{DA}$ in the range of $\nu=3000-3500$ $\mathrm{cm}^{-1}$, reflecting their deprotonation, the stretching and bending vibrations of the amine group (3340 and 1618 $\mathrm{cm}^{-1}$, respectively), and the stretching vibrations of $\mathrm{C}-\mathrm{H}$ $\left(3040 \mathrm{~cm}^{-1}\right)$ and $\mathrm{C}-\mathrm{C}$ of the aromatic ring $\left(1550 \mathrm{~cm}^{-1}\right)$. Moreover, the vibration band assigned to a $\mathrm{C}-\mathrm{O}$ stretch for the catechol coordinated to the metal appeared in the frequency range $1200-1300 \mathrm{~cm}^{-1}$. Additionally, typical vibrational bands of the BIX ligand $(\nu=1520,1262,1105$ $\mathrm{cm}^{-1}$ ) were observed in the FT-IR spectra. The ${ }^{1} \mathrm{H}$ NMR spectrum of DA-NCPs dissolved in a deuterated methanol$\mathrm{HCl}$ solution confirmed the presence of acetate counterions (see Supporting Information, Figure S5) and both ligands, although with a lower contribution of the BIX ligand than theoretically expected from the concentrations of the initial reactants. These results were quantitatively confirmed by highperformance liquid chromatography (HPLC) analysis of the product resulting upon treatment of DA-NCPs with an acidic solution; these analyses showed a content of $20 \%$ BIX $(w / w)$ ligand. More interestingly, HPLC analysis using electrochemical detection (HPLC-ECD) confirmed the presence of $52.5 \pm 7.2 \%$ DA in DA-NCPs nanoparticles (see Supporting Information and Materials and Methods section for experimental details). This exceptionally high value was confirmed with absorbance measurements using DA hydrochloride as a standard (Supporting Information, Figure S6). These analyses also revealed a very high loading efficiency (LE) of $69.7 \pm 7.2 \%$. For instance, this value is 6 -fold higher compared with the LE of dopamine achieved with PLGA in previous reports. As far as iron is concerned, inductively coupled plasma-mass spectroscopy (ICP-MS) data confirmed a total amount of $10.2 \%$ in the nanoparticles. Finally, the combination of elemental analysis, ICP-MS, ${ }^{1} \mathrm{H}$ NMR, and HPLC data yielded the following final tentative chemical formula for the nanoparticles: [Fe $\left.(\mathrm{DA})_{1.6}(\mathrm{BIX})_{0.5}(\mathrm{AcO})_{0.2}\left(\mathrm{H}_{2} \mathrm{O}\right)_{1.9}\right]$ (see Supporting Information, Table $\mathrm{S} 1$ ). This formula differs from that theoretically expected on the basis of the initial reagents added ( $1 \mathrm{Fe}: 1$ BIX:2 DA), which is attributed to their synthesis under out-ofequilibrium conditions induced by the fast precipitation process, a common phenomenon for this family of amorphous NCPs. Another plausible explanation for such differences could be the encapsulation of free ligand/solvent molecules within the particles present in the reaction solution, as already demonstrated in related systems. ${ }^{44,45}$ Such encapsulation ability has been confirmed by encapsulating, as a proof-ofconcept, the recombinant epidermal growth factor (EGF) with an excellent encapsulation efficiency (EE) of $89.6 \%$, and loading capacity (LC) of almost $7.7 \mu \mathrm{g}$ of EGF per milligram of particles (Supporting Information, Materials and Methods and Figure S7).

Colloidal Stability and DA Release Kinetics under Physiological Conditions. To simulate the colloidal stability of DA-NCPs in physiological environments, the nanoparticles were dispersed in water and phosphate-buffered saline (PBS) solution (with and without bovine serum albumin, BSA) at physiological conditions $(0.5 \mathrm{mM}) .{ }^{46,47}$ DLS measurements in water (Supporting Information, Table S2) showed higher hydrodynamic diameters (171 $\pm 4.2 \mathrm{~nm}$; PDI: 0.208) than those obtained in ethanol $(81 \pm 4.0 \mathrm{~nm}$; PDI: 0.124$)$ or as a solid powder by SEM measurements $(64 \pm 9 \mathrm{~nm})$. Moreover, even though the $\xi$-potential value in water was close to +25 $\mathrm{mV}$, the colloidal solution was not stable and started to aggregate and precipitate over a relatively short period of time (sedimentation observed after $1 \mathrm{~h}$ ). In PBS (20 mM PBS buffer at $\mathrm{pH} 7.4)$, the mean size values for nanoparticles indicated the presence of large aggregates (Supporting Information, Table S2) and showed a $\xi$-potential of -13.1 $\mathrm{mV}$. The addition of $0.5 \mathrm{mM}$ BSA, extensively used for dispersing inorganic and polymeric nanoparticles, ${ }^{48,49}$ successfully induced a disaggregation process and their stabilization without precipitation for several hours. The nanoparticles now showed a lower hydrodynamic diameter $(103 \pm 16.2 \mathrm{~nm}$; PDI: 0.198), similar to that observed using culture medium containing fetal bovine serum (FBS) (Supporting Information, Table S2). The increase in size $(24-28 \mathrm{~nm})$ with respect to the value found in ethanol could be attributed to the formation of a protein corona around the nanoparticles.

We next investigated the release kinetics of DA from DANCPs nanoparticles after incubation for $24 \mathrm{~h}$ in a PBS-BSA buffer at $37{ }^{\circ} \mathrm{C}$, using HPLC-ECD. At $\mathrm{pH} \mathrm{7.4,} \mathrm{a} \mathrm{continuous}$ release of DA, reminiscent of a standard saturation curve (Supporting Information, Figure S8), was observed with a cumulative release of $50 \%$ in the first $2 \mathrm{~h}\left(t_{1 / 2}=2 \mathrm{~h}\right)$. Afterward, the release notably slowed down with an increase of $5 \%$ in the next $22 \mathrm{~h}$. If the nanoparticles were dispersed at $\mathrm{pH} 5.5$, the accumulative release of $50 \%$ was reached in less than $1 \mathrm{~h}$. These results confirmed that the $\mathrm{pH}$ decrease induced a much faster release of the DA due to the lower stability of the coordinative bond between iron and catechol ligands. Finally, the release increased up to $85 \%$ in $10 \mathrm{~h}$ and $90 \%$ at $24 \mathrm{~h}$. This $\mathrm{pH}$ dependence has been observed previously in mussel-inspired hydrogels with Fe-catechol complexes and in other $\mathrm{Fe}$-containing polymeric nanoparticles. $^{30,50}$

Worth mentioning is that the FBS used in the previous experiments was heat-inactivated and does not fully represent the real physiological conditions found in the blood and plasma under in vivo conditions. For this reason, we also performed an additional experiment to study the colloidal stability of the DA-NCPs in the presence of nontreated human plasma. For this, an aliquot of DA-NCPs was suspended in $1 \mathrm{~mL}$ of ethanol or in $1 \mathrm{~mL}$ of nontreated human plasma. Afterward, the hydrodynamic diameter of the nanoparticles was evaluated at different time points of incubation at $37{ }^{\circ} \mathrm{C}$. No relevant changes in the size distribution of the nanoparticles up to $6 \mathrm{~h}$ incubation under these conditions were detected. Only a small shift toward higher dimensions was observed, compatible with the progressive formation of the protein corona due to the high concentration of proteins in the human plasma. As expected, 


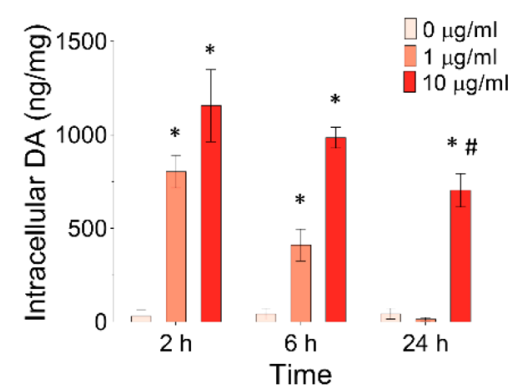

b

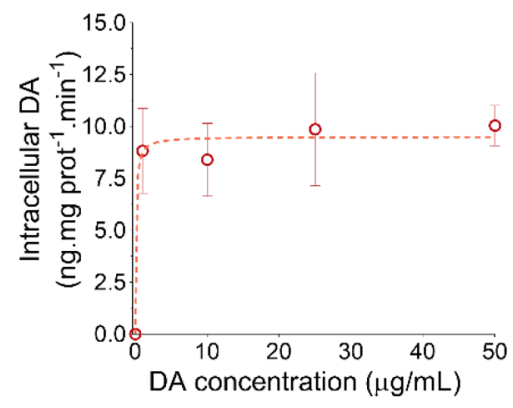

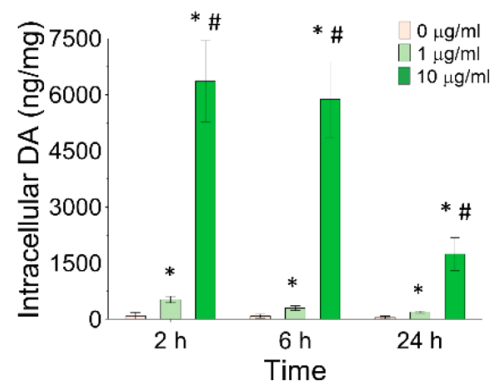

d

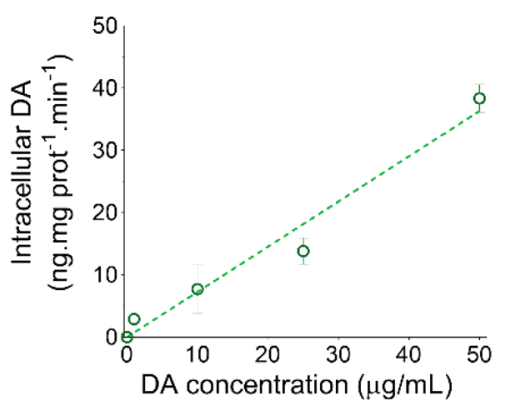

e
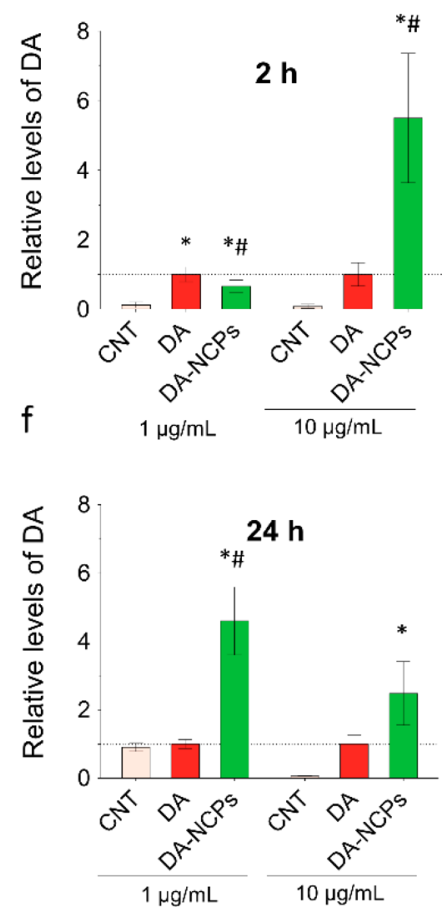

Figure 3. Uptake kinetics of DA and DA-NCPs by DArgic (BE)2-M17 cells. (a) Intracellular DA levels determined by HPLC-ECD after incubation of (BE)2-M17 cells for 2, 6, and $24 \mathrm{~h}$ in the absence $(0 \mu \mathrm{g} / \mathrm{mL}$, shown in light pink) or in the presence of DA at two different concentrations ( 1 and $10 \mu \mathrm{g} / \mathrm{mL}$ of DA, shown in light red and red, respectively). (b) Rate of DA uptake after the treatment of the cells with DA at 1, 10, 25, and $50 \mu \mathrm{g} / \mathrm{mL}$ for $2 \mathrm{~h}$. (c) Intracellular DA levels after incubation of (BE)2-M17 cells for 2, 6, and $24 \mathrm{~h}$ in the absence $(0 \mu \mathrm{g} / \mathrm{mL}$, shown in light pink) or in the presence of nanostructured DA (DA-NCPs) at two different concentrations (concentration equivalent to 1 and $10 \mu \mathrm{g} / \mathrm{mL}$ of DA, shown in light green and green, respectively). (d) Rate of DA uptake after the treatment of the cells with DA-NCPs at concentrations equivalent to DA concentrations of $1,10,25$, and $50 \mu \mathrm{g} / \mathrm{mL}$ for $2 \mathrm{~h}$. (e, f) Relative intracellular levels of DA after incubation for (e) $2 \mathrm{~h}$ and (f) $24 \mathrm{~h}$ in the absence $(0 \mu \mathrm{g} / \mathrm{mL}$, shown in light pink) or in the presence of DA or DA-NCPs at two different concentrations (concentration equivalent to 1 and $10 \mu \mathrm{g} / \mathrm{mL}$ ). In all cases, values are mean \pm SEM. In (a) and (c), $* p<0.05$, compared to $0 \mu \mathrm{g} / \mathrm{mL} ;{ }^{\#} p<0.05$, compared to treatment with $1 \mu \mathrm{g} / \mathrm{mL}$; a three-way ANOVA (full factorial) and Sidak correction was applied to the log-transformed data. In (e) and (f), ${ }^{*} p<0.05$, compared to control; ${ }^{*} p<0.05$, compared to DA (one-way ANOVA and Turkey's posthoc test).

the presence of these proteins was visible as a secondary peak (peak 2, in Figure S9) with a size of $\sim 40 \mathrm{~nm}$.

Evaluation of the Cytotoxicity of DA-NCPs against DArgic Neurons. To evaluate the effect of DA-NCPs on DArgic cell survival, in vitro cytotoxicity assays with (BE)2M17 cells were performed. (BE)2-M17 is a neuroblastomaderived DArgic cell line that expresses large amounts of key proteins essential for the DArgic metabolism and, therefore, extensively used as a cellular model to study DA metabolism. $^{51}$ Treatment of the (BE)2-M17 cells with DANCPs did not lead to any appreciable cell viability reduction after $48 \mathrm{~h}$ of incubation at concentrations up to $100 \mu \mathrm{g} / \mathrm{mL}$ (expressed as DA concentration) (see Supporting Information, Figure S10). The same concentrations of free DA in the range from 0.01 to $100 \mu \mathrm{g} / \mathrm{mL}$ showed comparable results. However, slightly higher levels of the free neurotransmitter (i.e., 200 and $250 \mu \mathrm{g} / \mathrm{mL}$ ) caused a dramatic death of DArgic cells with more than $85 \%$ reduction in the cell viability (Supporting Information, Figure S10). Interestingly, comparable concentrations of DA-NCPs caused lower cytotoxic effects. The toxicity found for the highest concentrations of DA was attributed to the intracellular metabolism of DA (conversion of DA to 3,4-dihydroxyphenylacetaldehyde, DOPAL) that generates hydrogen peroxide and, therefore, reactive oxygen species (ROS) effects. This process can take place both enzymatically (monoamine oxidase, MAO) or nonenzymatically. ROS can also be generated upon DA oxidation; when free DA cytosol levels exceed a certain threshold level, DA can spontaneously autoxidize, producing $o$-quinone molecules. ${ }^{52}$ These species are very reactive and may contribute to increasing the levels of oxidative stress inside the cells. To confirm this fact, intracellular ROS formation in $\mathrm{BE}(2)-\mathrm{M} 17$ cells was also measured after treatment with DA or DA-NCPs, through the $2^{\prime}, 7^{\prime}$ dichlorofluorescein diacetate (DCFDA) assay (see Materials and Methods). ${ }^{53}$ After a $24 \mathrm{~h}$ treatment, the 300 -fold DCF fluorescence increase found for the highest free DA concentration (see Supporting Information, Figure S11) confirmed the ROS production. ${ }^{24}$ Ascorbic acid (AA), at physiological concentrations of $20 \mu \mathrm{g} / \mathrm{mL}$, was required to drastically reduce ROS effects of $\mathrm{H}_{2} \mathrm{O}_{2}$. Interestingly, DANCPs showed a lower DCF fluorescence increase (200-fold) even considering the possible oxidative stress coming from iron ions (Supporting Information, Figure S12).

On the basis of these findings, we can state that our nanoparticles did not show a reduction in cell viability at the assayed DA concentrations in the range 0.01 to $10 \mu \mathrm{g} / \mathrm{mL}^{54}$ and that DA nanostructuring into DA-NCPs reduced the toxicity of the monoamine against DArgic cells. Similar observations were reported by Pahuja et al., ${ }^{24}$ who 
a

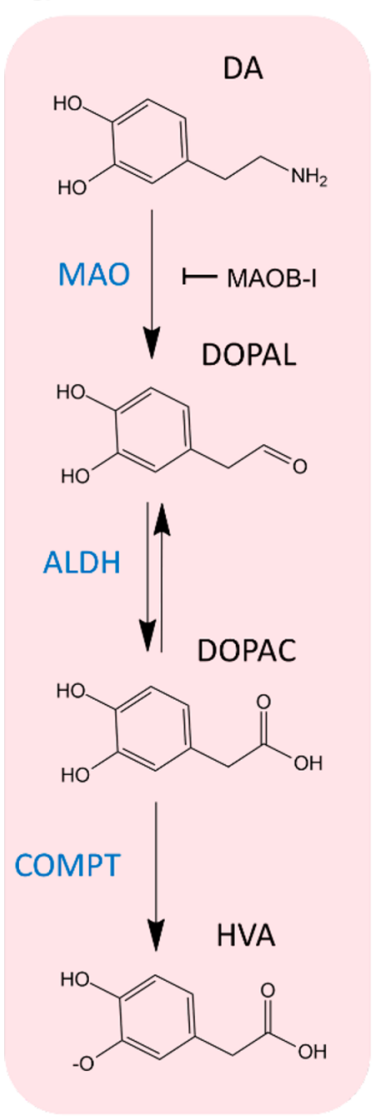

b
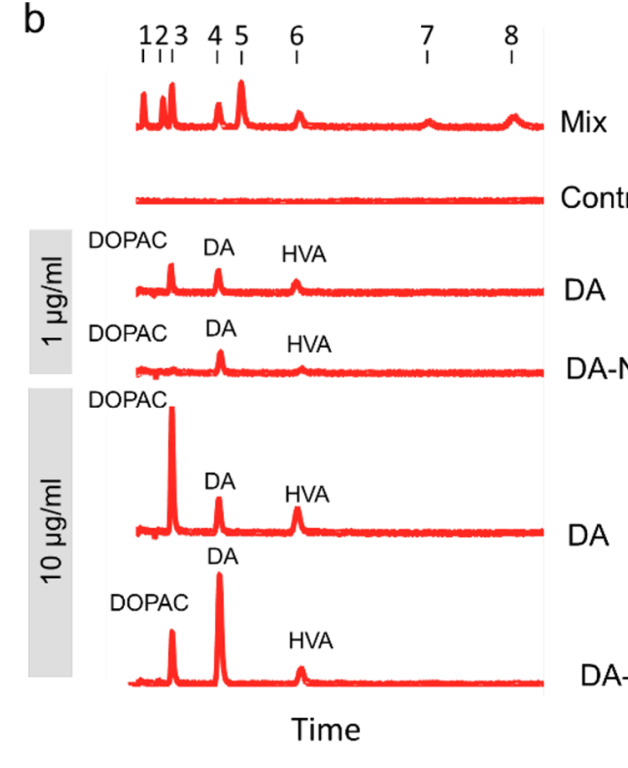

e

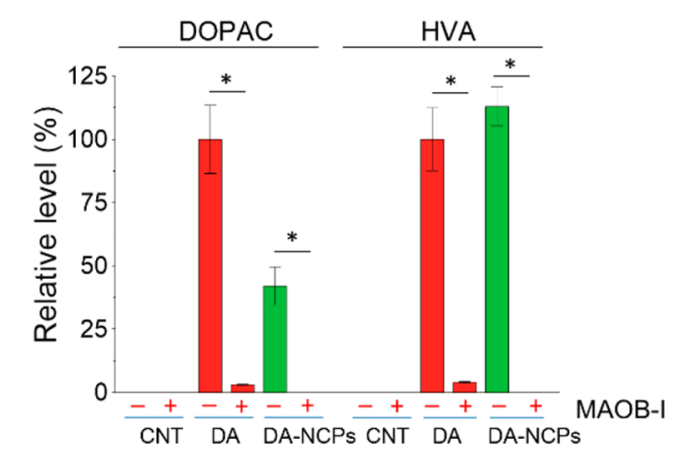

C

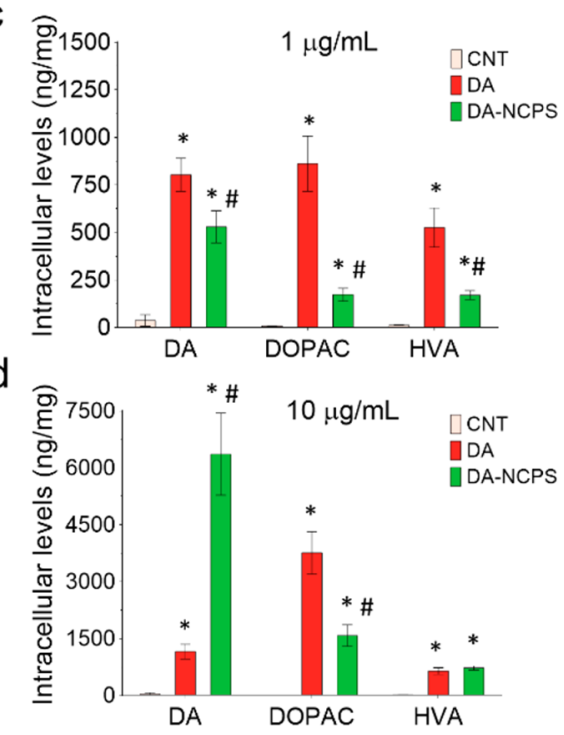

f

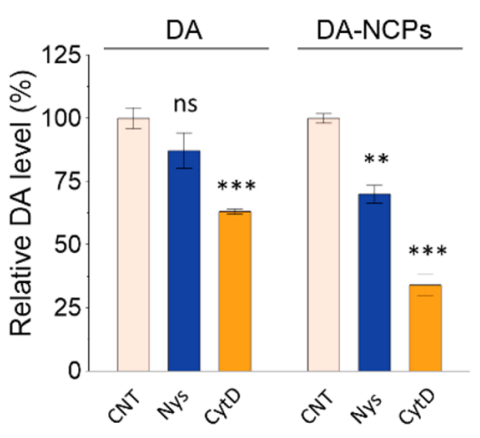

Figure 4. Metabolism kinetics of DA and DA-NCPs in DArgic neurons. (a) Principal degradation pathways of DA in DArgic neurons and main products obtained. (b) Representative HPLC-ECD chromatograms showing those DA derivatives found in (BE)2-M17 cells after treatment with two different concentrations $(1$ and $10 \mu \mathrm{g} / \mathrm{mL})$ of free DA or DA-NCPs for $2 \mathrm{~h}$. The mix of standards (mix) contained 1: $\mathrm{L}-$ DOPA; 2: DHBA, 3: DOPAC; 4: DA; 5: 5HIIA; 6: HVA, 7: 3-MT, and 8: 5HT. (c, d) Intracellular levels of DA and its metabolites (DOPAC and HVA) after $2 \mathrm{~h}$ of incubation with (c) $1 \mu \mathrm{g} / \mathrm{mL}$ or (d) $10 \mu \mathrm{g} / \mathrm{mL}$ of free DA or DA-NCPs. (e) Effect of deprenyl R, a specific monoamine oxidase inhibitor (MAOB-I), on DA metabolism. (f) Effects of chemical inhibition for selected endocytic pathways on DA uptake. Nys: nystatin; CytD: cytochalasin D. In all cases, values are mean \pm SEM and $n=6$ independent experiments, unless otherwise specified. In (c) and (d), * $p<0.05$, compared to CNT; ${ }^{*} p<0.05$, compared to treatment with DA (three-way ANOVA (full factorial) and Sidak correction was applied to the log-transformed data). In (e), $*_{p}<0.0001$, when compared between conditions with and without MAOB-I (two-tailed $t$-test). In (f), $* * p<0.01, * * * p<0.001$, ns (nonsignificant), when compared to CNT (two-tailed $t$-test); $n=3$ independent experiments.

demonstrated a reduction in the toxicity and oxidative damage induction of L-DOPA upon encapsulation into PLGA nanoparticles. Evaluation of the toxicity of the iron salt and the linker ligand was performed in (BE)2-M17 cell culture using concentrations ranging from 0 to $100 \mu \mathrm{g} / \mathrm{mL}$ for comparison purposes (see Supporting Information, Figure S13), with no cytotoxicity detected even at the highest concentration assayed $(100 \mu \mathrm{g} / \mathrm{mL})$. Finally, and for comparison purposes, we also studied the cytotoxicity of DA and DA-NCPs in a non-DArgic cell line such as HeLa cells (Supporting Information, Figure S13), where as expected, $\mathrm{IC}_{50}$ was smaller for both cases (see Supporting Information, Table S3).

Uptake of DA-NCPs by DArgic (BE)2-M17 Cells. The DA-NCPs' uptake kinetics by DArgic (BE)2-M17 cells was studied and compared to free DA. To perform the experiments, cells were exposed to increasing concentrations of DA or DA-NCPs and incubated for 2, 6, and $24 \mathrm{~h}$. Immediately after incubation, cells were collected and the intracellular concentration of DA was measured by HPLCECD (for more details, see Supporting Information and Materials and Methods).

Free DA. Incubation of (BE)2-M17 cells in the presence of $1 \mu \mathrm{g} / \mathrm{mL}$ of free DA resulted in a rapid concentrationdependent increase in intracellular DA level after $2 \mathrm{~h}$ of incubation (Figure 3a). This fact could be attributed to a large amount of DA transporters (DATs) that DArgic neurons use to control extracellular DA levels by pumping the extracellular DA from the synaptic cleft to the cytosol. ${ }^{55}$ As shown in Figure 3a, at longer incubations times (6 and $24 \mathrm{~h}$ ), a progressive decrease in the total intracellular DA concentration was observed. This decrease was accompanied by a constant reduction in the extracellular DA levels (see Supporting Information, Figure S14). The rate of uptake of DA through DAT is assumed to follow characteristic saturation kinetics. ${ }^{56}$ To confirm this, the experimental data obtained for the different DA concentrations used at $2 \mathrm{~h}$ were plotted and precisely fitted to Michaelis-Menten kinetics, 


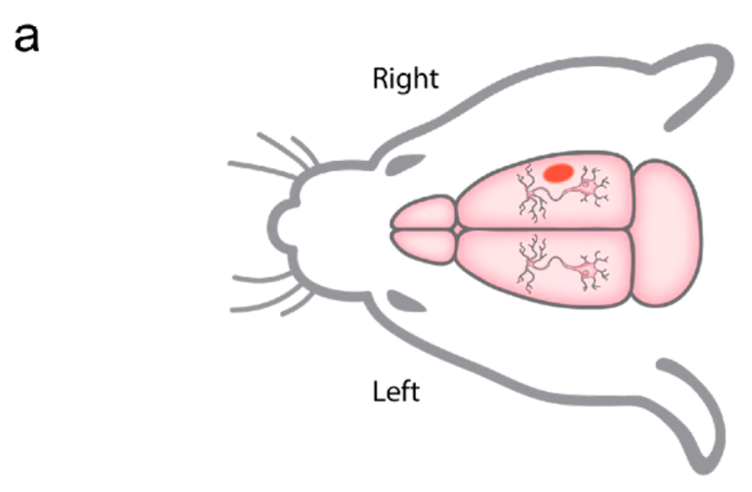

b

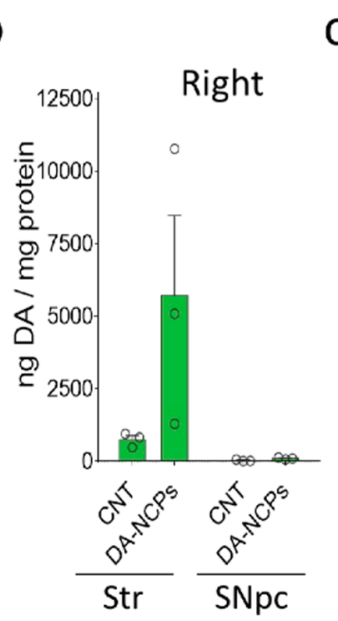

C

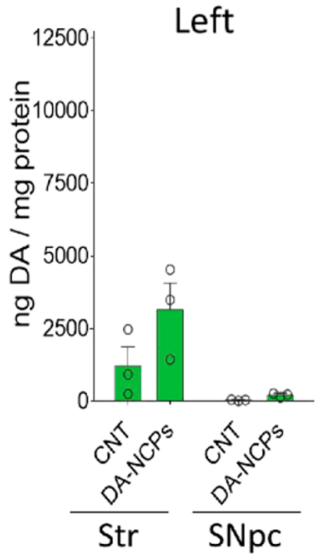

d

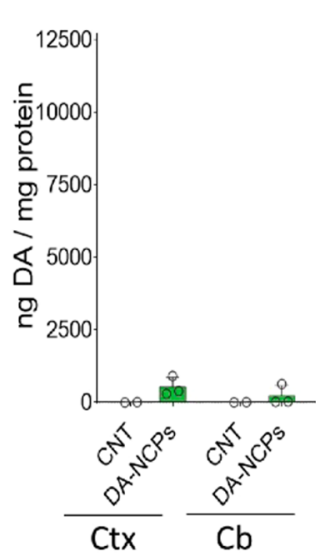

e Right

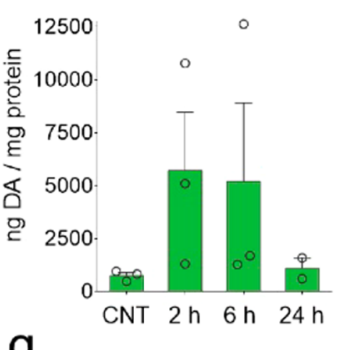

g

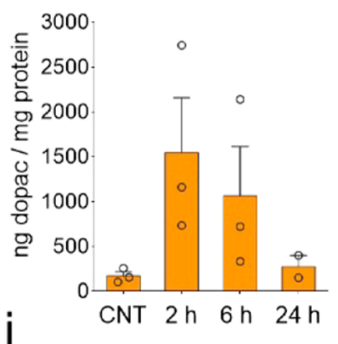

i

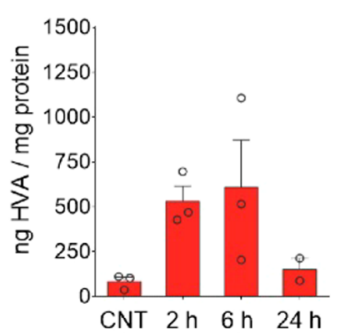

f

Left
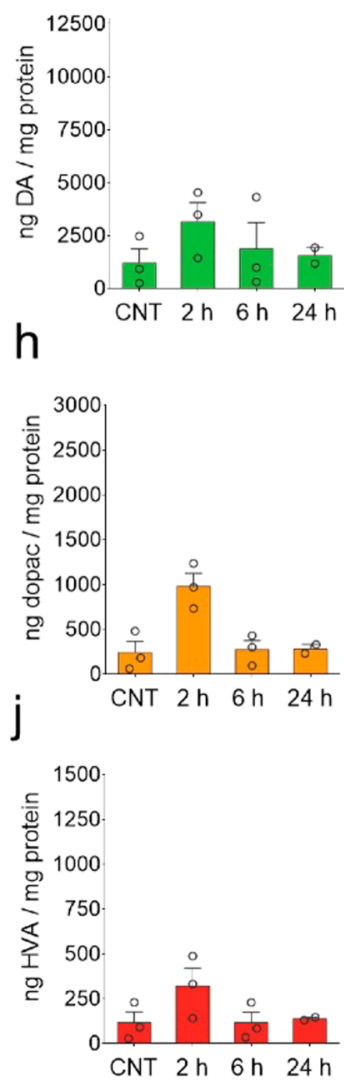

Figure 5. Biodistribution and metabolism of DA-NCPs in the brain after i.c.v. injection. (a) Schematic representation of the rat brain depicting the DA-NCPs injection site (red dot) and the location of the different regions analyzed (vide infra). (b, c) DA levels in the Str and SNpc (b) ipsilateral (right) or (c) contralateral (left) to the injected side after $2 \mathrm{~h}$ post-DA-NCPs administration. (d) DA levels in the $\mathrm{Ctx}$ and $\mathrm{Cb}$ after $2 \mathrm{~h}$ post-DA-NCPs injection. (e-j) Time-dependent metabolism of DA-NCPs after the i.c.v. injection of the nanoparticles. (e and $f$ ) DA, ( $g$ and $h$ ) dihydroxyphenylacetic acid (DOPAC), and ( $i$ and $j$ ) homovanillic acid (HVA) levels determined in the right and left striatum at different time points post-DA-NCPs injection. Data represent mean \pm SEM. In all cases $n=3$, unless otherwise indicated.

with approximate $K_{\mathrm{m}}$ and $V_{\max }$ values of $0.1 \mu \mathrm{g} / \mathrm{mL}$ and 10.0 $\mathrm{ng} \cdot \mathrm{mg} \operatorname{prot}^{-1} \cdot \mathrm{min}^{-1}$, respectively (Figure $3 \mathrm{~b}$ ). All in all, the results suggested that DA was actively taken up and metabolized by the DArgic (BE)2-M17 cells due to the action of the intracellular DArgic mechanism.

$D A-N C P$ s. Incubation of cells with DA-NCPs accounting overall for the same concentration of DA showed different intracellular profiles (Figure 3c,d). At $2 \mathrm{~h}$ and the lowest concentration $(1 \mu \mathrm{g} / \mathrm{mL})$, the DA internalized amount was slightly lower than that of free DA. Incubation of cells with higher doses $(10 \mu \mathrm{g} / \mathrm{mL})$ for the same period of time induced an intracellular level of DA up to $6360 \mathrm{ng} / \mathrm{mg}$. This concentration was 6-fold higher than that obtained for free DA (1156 ng/mg) under the same concentration and experimental conditions. Moreover, the plot of DA internalization $v s$ the initial external concentration used showed a nonsaturable dose-response mechanism instead of the characteristic Michaelis-Menten kinetics (Figure 3d), suggesting the presence of another pathway for nanoparticle uptake. Similar results and differences were found at $6 \mathrm{~h}$ of incubation, in agreement with the presence of DA outside the cells at this incubation time (Figure S14).

Note that the total intracellular DA determined by HPLCECD represents the sum of vesicular DA and the fraction of
DA present in the cytoplasm. In fact, only the excess of DA accumulated in the cytoplasm of the cells is available and can be degraded by the MAO. Therefore, the overall effect is a progressive and slow decline of intracellular DA levels that do not fully correspond to the extracellular DA concentration. Finally, after $24 \mathrm{~h}$ of incubation in the presence of $1 \mu \mathrm{g} / \mathrm{mL}$, DA-NCPs-exposed cells displayed a 4-fold amount of internalization compared to control and free DA-treated cells (Figure $3 \mathrm{a}$ and $\mathrm{f}$ ), where depletion of the extracellular DA caused normalization of the intracellular levels of the neurotransmitter. This finding is of particular significance as demonstrated by the enhanced intracellular retention of the neurotransmitter for longer periods of time upon nanostructuring.

Metabolism Kinetics of DA and DA-NCPs by DArgic Cells. The death of DArgic neurons in the substantia nigra leads to deficient striatal DA levels responsible for the cardinal motor symptoms of PD (i.e., including bradykinesia, tremor, and rigidity). ${ }^{57}$ Under normal conditions, the stimulation of healthy DArgic neurons causes the release of the synaptic vesicles into the synaptic cleft, where the neurotransmitter interacts with the postsynaptic DA receptors or regulatory presynaptic DA autoreceptors. ${ }^{58-62}$ When DA reaches the cytoplasm of DArgic neurons, this neurotransmitter is rapidly 
a)

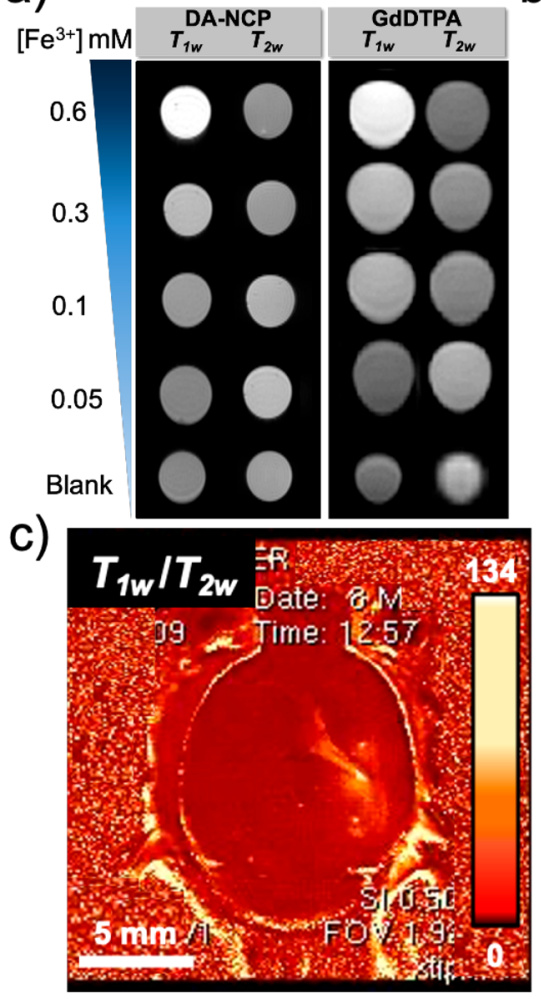

b)

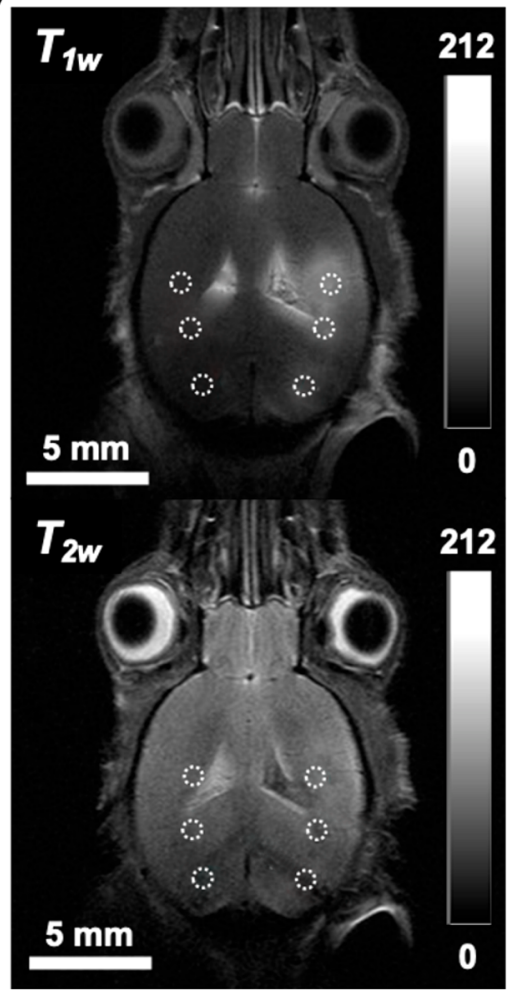

Figure 6. Magnetic resonance imaging (MRI) experiments. (a) In vitro $T_{1 \mathrm{w}}$ and $T_{2 \mathrm{w}}$ MRI of phantoms with DA-NCPs in comparison with commercial GdDTPA at decreasing metal concentration. (b) Ex vivo DA-NCPs $T_{1 \mathrm{w}}$ (top) and $T_{2 \mathrm{w}}$ (bottom) MRI obtained after stereotactic injection of DA-NCPs. (c) Postprocessing of $T_{1 \mathrm{w}}$ and $T_{2 \mathrm{w}}$ images by an algebraic algorithm of imaging division $\left(T_{1 \mathrm{w}} / T_{2 \mathrm{w}}\right)$. For the ex vivo experiments, DA-NCPs and GdDTPA were stereotactically injected in brain parenchyma. The dashed circles indicate the selection of ROIs for the RCE calculation. Color scales show the range of pixel values.

stored back into the synaptic DA storage vesicles. However, an excess of DA in the cytoplasm of the cell is metabolized into 3,4-dihydroxyphenylacetic acid (DOPAC) by monoamine oxidase $\mathrm{B}$ enzyme (MAO-B) $)^{58}$ and aldehyde dehydrogenase $(\mathrm{ALDH})$. Then DOPAC is converted into homovanillic acid (HVA) by the action of catechol-O-methyltransferase (COMT) (see Figure 4a). ${ }^{59}$ Besides, synaptic cleft DA can be taken up by surrounding glial cells and be degraded by MAO and also by COMT, which transforms DOPAC into HVA, one of the main degradation products of DA (Figure 4a).

To study this metabolism and degradation kinetics, the intracellular concentration of DA and derived metabolites was determined using HPLC-ECD in (BE)2-M17 cells incubated with free DA and DA-NCPs for $2 \mathrm{~h}$ and at two concentrations ( 1 and $10 \mu \mathrm{g} / \mathrm{mL}$ ). As shown in Figure $4 \mathrm{~b}$, in both cases levels of DA and metabolites increased though DOPAC and HVA levels were 5.3 times and 3.1 times, respectively, lower for DA-NCPs than for free DA (Figure $4 b, c$ ). In other words, at $1 \mu \mathrm{g} / \mathrm{mL}$ the $\mathrm{DA} /(\mathrm{DOPAC}+\mathrm{HVA})$ ratios for free DA or DA-NCPs were 0.58 and 1.54 , respectively ( $\sim 2.6$-fold reduction for DA-NCPs). This difference was accentuated at $10 \mu \mathrm{g} / \mathrm{mL}$ (Figure 4d), where the DA/(DOPAC+HVA) ratios were 0.30 and 2.80 for DA and DA-NCPs, respectively (9.3fold increase).

As anticipated, free DA exhibited a progressive reduction in DOPAC and HVA levels with time for the $1 \mu \mathrm{g} / \mathrm{mL}$ concentration (see Supporting Information, Figure S15), while a higher DOPAC production and less abrupt reduction were observed at $10 \mu \mathrm{g} / \mathrm{mL}$ (HVA levels remained quite homogeneous with time). A similar tendency was found for DA-NCPs at the lowest concentration, though at higher concentrations $(10 \mu \mathrm{g} / \mathrm{mL})$ the generation of metabolites remained constant and only fell after $24 \mathrm{~h}$. These observations could be indicative of the DA-reservoir properties of DANCPs and a relatively slow release of free DA from the nanoformulation, which limits the formation of metabolites. Another essential factor to determine was how DA-NCPs (or DA released from them) could be metabolized by MAO-B. For this, we incubated (BE)2-M17 cells with the DA-NCPs in the presence of $2 \mu \mathrm{M}$ deprenyl-R, a specific monoamine oxidase B inhibitor (MAOB-I). As shown in Figure 4e, the levels of DOPAC and HVA fell following treatment with this particular chemical inhibitor. Similar results were observed in the case of free DA-treated cells, suggesting that DA released from the nanoparticles could be actively processed by the oxidative DArgic mechanism.

Finally, we evaluated the uptake of DA or DA-NCPs in the presence of specific inhibitors of endocytic pathways. As shown in Figure $4 \mathrm{f}$, the presence of cytochalasin D (CytD) induced a small decrease in DA uptake for free DA, while both nystatin (Nys) and CytD caused an important reduction in the uptake of DA-NCPs. These results indicated that an essential fraction of the internalization of DA-NCPs was mediated by specific endocytic pathways. Moreover, actin/ dynamin interactions play a central role in the recycling of DAT via the bulk endocytic route. In fact, treatment of adult dopaminergic neurons with $\mathrm{CytD}$ (used to disrupt the actin 
a

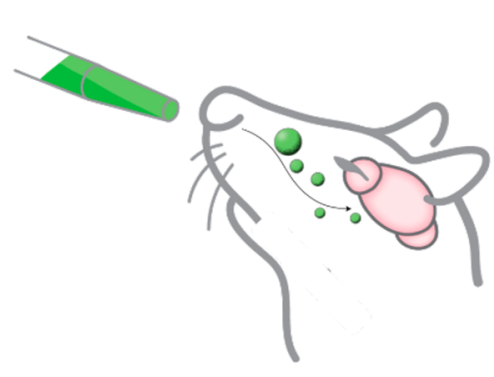

b

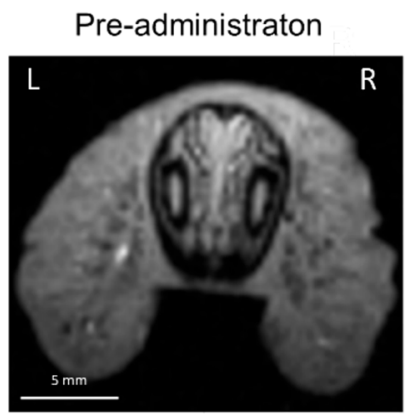

C

\section{Post-administraton}

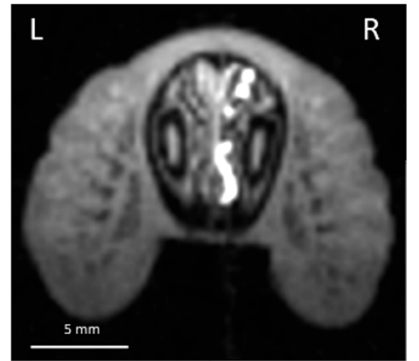

\section{f}

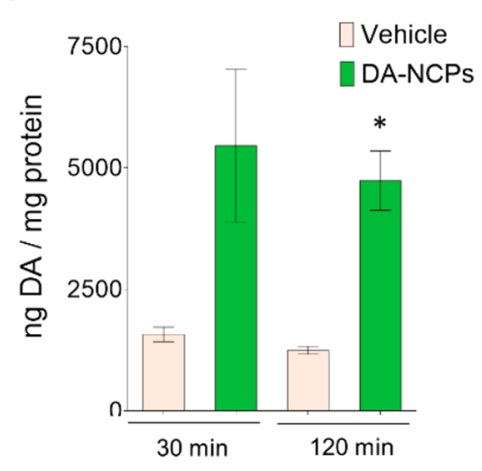

$\mathrm{h}$

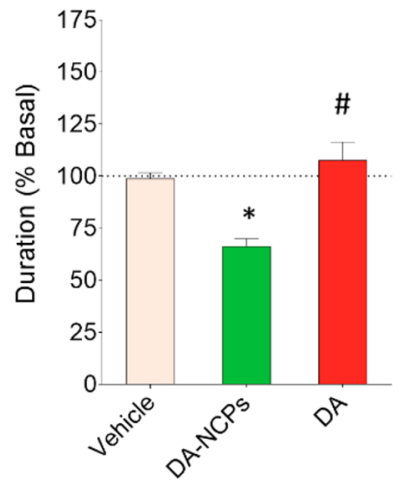

Figure 7. Intranasal administration of DA-NCPs and pharmacological effects on the 6-OHDA rat model of PD. (a) Schematic representation for the intranasal administration of the DA-NCPs. (b-f) Biodistribution of intranasally applied DA-NCPs. $T_{1 w}$ MRI from the nasal cavity of a rat obtained (b) before (pretreatment) and (c) after (post-treatment) intranasal application of the nanoparticles. (d) DA levels detected in the plasma of healthy rats treated with vehicle or with a dose of DA-NCPs equivalent to $50 \mu \mathrm{g}$ of free DA. (e) DA concentrations in the right striatum of healthy rats determined at two different time points $(30$ min and $2 \mathrm{~h})$ post-DA-NCP treatment. Values are expressed as $\mathrm{ng}$ of DA/mg of protein. Data are presented as mean \pm SEM. (f) Levels of DA in the right striatum of healthy rats. The test groups include animals treated with vehicle or intranasally administered with a dose of DA or DA-NCPs (dose equivalent to $50 \mu \mathrm{g}$ of DA). Values are presented as percentage of DA compared to the vehicle-treated animals. Data are presented as mean \pm SEM. (g) Apomorphine-induced rotational behavior of 6-OHDA-lesioned rats. Data are percentage of apomorphine-induced rotations determined 24 $h$ postadministration. The net number of contralateral rotations was calculated as total left $360^{\circ}-$ total right $360^{\circ}$ turns, and data are presented as percentage of rotations in comparison with values achieved in basal conditions before treatment. Duration of the apomorphine-induced rotational behavior. Data are expressed as percentage of the time achieved in the basal experiment before treatment. The apomorphine-induced rotation test was performed in 6-OHDA-lesioned rats previously treated with repeated administrations of vehicle $(n=6)$, DA $(n=6)$, or DA-NCPs $(n=6)$. During the semichronic treatment, each animal received a total dose of DA or DA-NCPs equivalent to $800 \mu \mathrm{g}$ of free DA, administered on 4 consecutive days $(200 \mu \mathrm{g} /$ day $)$. In all cases, values are shown as mean \pm SEM. In $(\mathrm{d})$ and (e) ns (nonsignificant) when compared to vehicle; *p< 0.05 , compared to vehicle (two-tailed $t$-test). In (f) $-(\mathrm{h})$, ns, *p $<0.05$, compared to vehicle; ${ }^{\#} p<0.05$, compared to treatment with DA-NCPs (one-way ANOVA and Tukey's posthoc test).

filaments) significantly reduces DAT surface levels to $69.9 \pm$ $5.1 \%$ of control levels. ${ }^{63}$ This reduction in the number of receptors on the surface of the cells is compatible with the decrease in the DA uptake $(63.1 \pm 2.8 \%$ compared to control) observed in our experiments upon CytD treatment. 
In Vivo Biodistribution and Metabolism of DA-NCPs within the Brain. To examine the in vivo brain biodistribution of DA-NCPs, a single dose was infused in the lateral ventricle of healthy adult male Sprague-Dawley rats (Figure 5a) through a surgical cannula installed in the right ventricle. The i.c.v. infusion of DA and DA agonist has been attempted in the past in rodents, primate models, and human patients of PD as an alternative to conventional treatments. ${ }^{64}$ Venna and co-workers reported a good tolerance to continuous i.c.v. DA infusion in patients treated over 1 year. ${ }^{65}$ This continuous DA delivery resulted in a more stable treatment effect without the "on-off" fluctuations of traditional oral therapy.

The materials were well tolerated by the animals, as no adverse effects nor increased mortality was observed during nanoparticle administration, in agreement with the low in vitro toxicity values and the absence of systemic toxicity previously reported. The animals were then euthanized to measure the DA and DA metabolite levels of different brain regions using HPLC-ECD at different time points after intracerebroventricular (i.c.v.) injection. As shown in Figure 5, distribution of DA-NCPs from the right lateral ventricle to the surrounding neuronal tissues occurred mostly within the first $2 \mathrm{~h}$, with a notable increase in DA levels observed in the striatum and $\mathrm{SNpc}$ (Figure 5b,c and Figure S16). This observation suggested that DA released from the nanoparticles was readily bioavailable and could be efficiently incorporated by nigrostriatal DArgic neurons in vivo.

It is worth mentioning that higher amounts of DA could be detected in the right striatum ipsilateral to the site of administration, in comparison with the levels detected for the left striatum. In addition to the nigrostriatal track, we identified small amounts of DA in other regions of the brain, such as the cortex or the cerebellum (Figure 5d). Striatal DA concentration recovered its basal levels after $24 \mathrm{~h}$ postinjection, indicating that DA-NCPs were completely cleared and/or metabolized in the brain by DArgic neurons or by surrounding glial cells (Figure 5e,f). Thus, we further investigated the DA-NCPs metabolism in the brain by measuring the levels of the two main metabolites, DOPAC and HVA. As shown in Figure 5g-j, DOPAC and HVA levels showed an increase in the right striatum after 2 and $6 \mathrm{~h}$ postadministration, in agreement with the in vitro metabolism experiments using DArgic cells.

DA-NCPs Display Magnetic Resonance Imaging Properties. Longitudinal $\left(r_{1}\right)$ and transversal $\left(r_{2}\right)$ relaxivity values were determined at $7 \mathrm{~T}$ by measuring corresponding relaxation rates in phantoms containing different concentrations of DA-NCPs dispersed in PBS/agarose $1 \%$ solution at $\mathrm{pH} 7.4\left(T_{1}\right.$ and $T_{2}$ map sequences, see detailed protocols in the Supporting Information; representations of $R_{1}, R_{2} v s$ metal concentration are shown in Supporting Information, Figure S17). As presented in Figure 6a, samples showed increased $T_{1 \mathrm{w}}$ enhancement (brighter signal) with the increase of the metal ion concentration. The $r_{1}$ value was determined to be $5.1 \pm 0.6 \mathrm{mM}^{-1} \mathrm{~s}^{-1}$ for DA-NCPs, which was comparable with related iron-based NCPs (i.e., $r_{1}=4.4 \mathrm{mM}^{-1} \mathrm{~s}^{-1}$ for FeNCPs), ${ }^{6,67}$ previously reported iron-based coordination polymers, ${ }^{68,69}$ paramagnetic molecular complexes $\left(3.0 \mathrm{mM}^{-1}\right.$ $\left.\mathrm{s}^{-1}\right){ }^{70}$ or even commercial gadolinium diethylenetriaminepentacetate (GdDTPA) contrast agents (CAs) (Magnevist, $4.4 \pm 0.2 \mathrm{mM}^{-1} \mathrm{~s}^{-1}$ ) (Figure 6b). Such an exceptional response could be directly related to the ability of catechol- based ligands to maximize second-sphere interactions with water molecules and therefore enhance the $T_{1 \mathrm{w}}$ MRI signal through hydrogen bonding with the oxygen atoms of the $\mathrm{Fe}-$ $\mathrm{O}-\mathrm{R}$ linkages. ${ }^{71}$ Moreover, DA-NCPs probably contained water molecules coordinated to the metal sites, as well as free water molecules, which probably exchange with surrounding water molecules, diffusing through the polymeric matrix. ${ }^{72}$ Encouraged by these exceptional results and considering that phantom results are not always fully comparable with contrast enhancement in real tissues, ${ }^{73-75}$ ex vivo studies with DANCPs were conducted following a methodology previously reported. ${ }^{76}$ For this, PBS-BSA dispersions of DA-NCPs and commercial GdDTPA (for comparison purposes) were intracranially administered post mortem to euthanized mice. $T_{1 \mathrm{w}}$ and $T_{2 \mathrm{w}}$ MRI was subsequently acquired, and relative contrast enhancement (RCE) calculated (see Table S4) (details about calculations and protocol can be found in the Supporting Information and Materials and Methods section).

Three different regions of interest (ROIs) were selected in $T_{1 \mathrm{w}}$ and $T_{2 \mathrm{w}}$ images, corresponding to the CA administration points (Figure $6 \mathrm{~b}$ ). DA-NCPs presented higher RCE $T_{1}$ in comparison with the commercial CA GdDTPA and comparable RCE $T_{2}$. The postprocessing of $T_{1 \mathrm{w}}$ and $T_{2 \mathrm{w}}$ images by an algebraic algorithm of imaging division $\left(T_{1 \mathrm{w}} /\right.$ $T_{2 \mathrm{w}}$ ) showed a clear brightness area when DA-NCPs were injected (Figure 6c) into the brain, confirming the excellent behavior of DA-NCPs for MRI monitoring.

Intranasal Administration of DA-NCPs. The direct infusion of DA-NCPs demonstrated rapid biodistribution and pharmacokinetic potential. However, this administration route is not an appropriate choice for the continued treatment of PD patients since it is invasive and requires a surgical procedure for the insertion of the i.c.v. device. So, the potential application of DA-NCPs for nose-to-brain delivery of DA was then evaluated (Figure 7a) in rats. First, we aimed to track the biological pathway followed by the nanoparticles after their intranasal administration using in vivo MRI. As shown in Figure $7 \mathrm{a}$ and $\mathrm{b}$, a strong $T_{1 \mathrm{w}}$ signal was clearly observed in the right nasal cavity of the rat, near the respiratory epithelia. $T_{1}$ values measured within ROIs from three consecutive sections were significantly decreased after different preadministration of DA-NCPs $\left(T_{1}=707 \pm 119 \mathrm{~ms}\right)$ compared to postadministration values $(1631 \pm 91 \mathrm{~ms}$, $t$-test: $p=0.003)$. Previous intranasal studies demonstrate that drugs gain direct access to the central nervous system (CNS) via direct pathways from the nasal cavity. ${ }^{77}$ A more detailed analysis of different longitudinal and coronal sections of the anterior rat head and nasal cavities (Figure S18a,b) elucidated the presence of a strong DA-NCPs signal in the right duct of the vomeronasal organ. These results clearly showed that DANCPs were in contact with the mucosa and the epithelia of the nasal cavity immediately before they were transferred into the CNS via the olfactory bulb. ${ }^{78}$

Plasma DA levels of healthy rats after $2 \mathrm{~h}$ of administration did not show relevant differences between samples taken from rats treated with DA-NCPs (dose equivalent to $50 \mu \mathrm{g}$ of free DA) or only with the vehicle (Figure $7 d$ ), indicating that DA was not transferred to systemic circulation after this time. By contrast, significantly higher amounts of striatal DA were detected in the ipsilateral side of the rat brains after $30 \mathrm{~min}$ and $2 \mathrm{~h}$ of unilateral DA-NCPs nasal administration (Figure $7 \mathrm{e})$. The maximum DA concentration was achieved after 30 min with a 3.5 -fold increase in comparison with basal striatal 
DA levels, consistent with observations noted in earlier intranasal investigations. ${ }^{78}$ Time evolution analysis of HVA and DOPAC metabolites in the same samples nicely correlated with that of DA, showing a clear increase of these two metabolites associated with the simultaneous activation of the DA catabolism in the right striatum. Interestingly, no significant increase in DA was observed in the contralateral striatum after unilateral administration of DA-NCPs (Figure S18c). Similar to DA-NCPs, unilateral intranasal administration of an equivalent dose of free DA into the right nostril also led to a clear increase of the striatal DA, reaching comparable levels to those observed in the case of the nanoparticles (Figure 7f).

Pharmacological Effect in 6-HydroxyDA (6-OHDA)Lesioned Rats. To determine if these increased levels of DA in the striatum of DA-NCPs-treated rats are physiologically relevant, we examined the pharmacological effect of the nanoparticles in 6-hydroxyDA (6-OHDA)-lesioned rats, a PD model characterized by a typical unilateral drug-induced degeneration of the nigrostriatal DArgic neurons on the same side as neurotoxin injection. Measurements following intraperitoneal injection of apomorphine, a potent DArgic agonist, in the 6-OHDA-lesioned rats revealed a characteristic contralateral turning behavior (average of rotations in all the groups analyzed in the basal test: $249 \pm 146$ contralateral turns; average duration of the rotational behavior: $57 \pm 11$ $\mathrm{min}$ ) in response to the stimulation of the DA receptors. After 4 days of nasal administrations with DA-NCPs equivalent to $200 \mu \mathrm{g}$ of the free drug per day, the number and duration of apomorphine-induced rotations were significantly lower from that in either vehicle- or DA-treated rats performed for comparison purposes (Figure $7 \mathrm{~g}, \mathrm{~h}$ ). This shortening in the motor response is compatible with the continuous presence of $\mathrm{DA}$ in the nigrostriatal pathway, indicating desensitization in unilateral 6-OHDA-lesioned rats continuously treated with the nanoparticles. Compared to DA-treated animals, the different therapeutic response observed in DA-NCPs might be attributed to their more sustained release potential.

\section{CONCLUSIONS}

Inspired by natural NM, we have reported a family of DAbased coordination polymer nanoparticles as stable colloidal suspensions with FBS or BSA and high encapsulation yields (close to 60\%). The encapsulated DA exhibited a reduced toxicity against DArgic cells by comparison with free DA and a decreased ROS production even with the incorporation of iron ions. In fact, the nanoparticles proved safe in vivo, with no significant weight loss or toxicity symptoms for the 30-day period after the last injection administration. The nanostructuring as DA-NCPs also favors (in comparison with the incubation of DArgic cells with free DA) enhanced intracellular retention of the neurotransmitter intracellularly released for extended periods of residence. This fact is mainly attributed to both a very effective incorporation of the nanoparticles by DArgic cells mediated by specific endocytic pathways and to slower metabolism kinetics into DA metabolites (i.e., DOPAC and HVA) due to the restrictions imposed by the DA supramolecular assembly. The i.c.v. infusion of the nanoparticles into the living brain of SpragueDawley rats was sufficient to promote an increase in striatal and SNpc DA levels as soon as $2 \mathrm{~h}$ after administration. DA metabolites also increased accordingly, suggesting an efficient clearance of the excess of DA in these living tissues.
Finally, we studied direct delivery of the nanoparticles to the brain via nasal administration. Our findings showed an efficient delivery of DA to the nigrostriatal pathway capable of attenuating motor alterations in a 6-OHDA-induced animal model of PD. On top of that, the presence of iron ions was used to track the nanoparticles by MRI after postprocessing of the $T_{1 \mathrm{w}}$ and $T_{2 \mathrm{w}}$ images. All in all, these results position this last generation of DA-based nanoparticles in the field of DA replacement therapy with nanoparticles.

\section{MATERIALS AND METHODS}

One-Pot Synthesis of DA-NCPs. DA-NCPs were prepared according to our previously published method for the synthesis of NCPs with some modifications. ${ }^{41,44,79}$ Briefly, a mixture of DA hydrochloride $(0.5 \mathrm{mmol}, 95 \mathrm{mg})$ and BIX $(0.25 \mathrm{mmol}, 60 \mathrm{mg})$ was dissolved in $12.5 \mathrm{~mL}$ of ethanol. Under constant magnetic stirring $(900 \mathrm{rpm})$, an ethanolic solution of $\mathrm{Fe}\left(\mathrm{CH}_{3} \mathrm{COO}\right)_{2}(0.25 \mathrm{mmol}, 44$ $\mathrm{mg}$ in $2.5 \mathrm{~mL}$ of ethanol) was added to the DA-BIX solution. The reaction mixture was stirred at room temperature for $2 \mathrm{~h}$, and the suspension rapidly turned from colorless to black, which indicates the formation of a fine precipitate. After $2 \mathrm{~h}$, the precipitate was centrifuged at $8000 \mathrm{rpm}$ for $10 \mathrm{~min}$. The pellet obtained was resuspended and washed three times with ethanol. The sample was divided into two batches; in one of them, the solvent was removed and the solid dried under vacuum and fully characterized by UV-vis, FT-IR, ICP-MS, elemental analysis, ${ }^{1} \mathrm{H}$ NMR, and HPLC. The remaining batch was kept under solution to avoid problems with aggregation of the sample during the drying procedure and was characterized by DLS, zeta potential, TEM/STEM, and HPLC. For in vivo experiments, the final pellet containing the nanoparticles was resuspended in ethanol (to reach a final DA-NCPs concentration of $\geq 20.0 \mathrm{mg} / \mathrm{mL}(\mathrm{w} / \mathrm{v}))$ and stored under solution at $4{ }^{\circ} \mathrm{C}$ until use.

Size and Zeta-Potential Measurements. Mean particle size and zeta potential of the DA-NCPs were determined by DLS using a Zetasizer Nano 3600 instrument (Malvern Instruments, UK). Samples of the nanoparticles were dispersed in five different solvents: ethanol absolute, $70 \%$ ethanol, PBS, PBS with $30 \mathrm{mg} / \mathrm{mL}(\sim 0.5$ $\mathrm{mM}$ ) of BSA, or Opti-MEM culture medium containing $10 \%$ heatinactivated FBS. In order to study the physical stability of DA-NCPs over time, an aliquot of nanoparticles was dispersed in $70 \%$ ethanol, PBS-BSA, or culture medium with FBS, and each suspension was analyzed immediately or after $10,30,60$, and 120 min of incubation at $25{ }^{\circ} \mathrm{C}$. In all cases, the nanoparticles were dispersed at a concentration of $100 \mu \mathrm{g} / \mathrm{mL}$. Measurements were performed in automatic mode, as an average of 14-24 runs and in triplicate.

TEM and EDX Analysis. Samples of DA-NCPs were suspended in ethanol, diluted to $200 \mu \mathrm{g} / \mathrm{mL}$, and placed onto carbon-coated grids. After $2 \mathrm{~min}$, grids were dried carefully and negatively stained with a $2 \%(\mathrm{w} / \mathrm{v})$ uranyl acetate solution for $2 \mathrm{~min}$. Micrographs were acquired under a Hitachi H-7000 TEM with a $75 \mathrm{kV}$ accelerating voltage. The diameter of the particles was measured using ImageJ software. $^{80}$ For EDX analysis, DA-NCPs were diluted in ethanol, layered over carbon-coated grids, and then preparations washed twice with distilled water. An EDX spectrum of the nonstained nanoparticles was obtained in a Jeol JEM-1400 microscope operating at $120 \mathrm{kV}$, by collecting X-rays emitted from the local volume probed by the electron beam.

SEM Analysis. The structural properties (shape, surface morphology, and particle size distribution) of the DA-NCPs were studied by a high-resolution field emission SEM in a Hitachi S-570 SEM. Samples of nanoparticles were diluted in ethanol and placed over an aluminum tape. Following solvent evaporation, samples were coated with platinum under an argon atmosphere by using an Emitech K550 sputter coater (Emitech Ltd., Ashford, Kent, UK). SEM micrographs were acquired at an accelerating voltage of $15 \mathrm{kV}$ under a high-vacuum mode and a distance of the sample of $5 \mathrm{~mm}$.

UV-Vis Spectroscopy and Spectroscopic DA Quantitation. The absorbance spectra of DA-NCPs, as well as spectra of its 
polymeric precursors (DA, and BIX), were recorded using a Cary 4000 spectrophotometer (Agilent), in the $200-800 \mathrm{~nm}$ wavelength range, and using a matched pair of quartz cuvettes of $1 \mathrm{~cm}$ optical path length. Immediately before analysis, samples were diluted in filtered Milli-Q water to a final concentration of $100 \mu \mathrm{g} / \mathrm{mL}$. For the spectroscopic DA quantitation, $5 \mathrm{mg}$ of dried DA-NCPs was dissolved in $5 \mathrm{~mL}$ of $1 \mathrm{M} \mathrm{HCl}$, sonicated until the complete dissolution of the nanoparticles, and diluted up to a concentration of $0.1 \mathrm{mg} / \mathrm{mL}$ in $1 \mathrm{M} \mathrm{HCl}$. Standards with different DA concentrations (ranging from 0 to $0.4 \mathrm{mM}$ ) were prepared by diluting a stock solution $(1 \mathrm{mM})$ of DA hydrochloride in $1 \mathrm{M} \mathrm{HCl}$ solution. The absorbance of the different samples was determined at $278 \mathrm{~nm}$ using quartz cuvettes ( $1 \mathrm{~cm}$ optical path length) in a Cary 4000 spectrophotometer (Agilent). The amount of loaded DA was calculated from the calibration curve (adjusted to a linear regression model with $R^{2}>0.99$ ) (see Supporting Information, Figure S6). The loading efficiency was determined using the following equation: LE in DA-NCPs $(\%)=\left(\right.$ Weight $\left._{\mathrm{DA}}\right) /\left(\right.$ Weight $\left._{\mathrm{NCPs}}\right) \times 100$.

FT-IR Spectroscopy. FT-IR spectra of the nanoparticles and precursors were recorded by transmission FT-IR on a Bruker Tensor 27 (Bruker Optics) spectrophotometer. Pellets were prepared by mixing approximately $1 \mathrm{mg}$ of the test sample with $100 \mathrm{mg}$ of $\mathrm{KBr}$, followed by subjection to high pressure using a manual pellet marker. Each spectrum was acquired between 400 and $4000 \mathrm{~cm}^{-1}$ at a spectral resolution of $2 \mathrm{~cm}^{-1}$.

${ }^{1} \mathrm{H}$ NMR Measurements. The chemical composition of DANCPs was further investigated by ${ }^{1} \mathrm{H}$ NMR. In a typical experiment, $5 \mathrm{mg}$ of dry DA-NCPs were dispersed in deuterated methanol. To ensure complete dissolution of the nanoparticles and the liberation of the ligands by demetalation, two drops of deuterated hydrochloric acid were added to the suspension. After incubation for $30 \mathrm{~min}$ at 25 ${ }^{\circ} \mathrm{C},{ }^{1} \mathrm{H}$ NMR spectra were recorded using a Bruker Avance DRX 250 NMR spectrometer equipped with a $5 \mathrm{~mm}$ broad band inverse probe-head working at $250 \mathrm{MHz}$ for ${ }^{1} \mathrm{H}$. Chemical shifts were referenced to the trace signals of acetone. The reference sample was prepared by mixing $10 \mathrm{~mL}$ of acetone into $0.5 \mathrm{~mL}$ of deuterated methanol in a WILMAD coaxial insert tube, which was immersed in the $5 \mathrm{~mm}$ NMR sample tube (acetone chemical shift: $2.15 \mathrm{ppm}$ ). As a reference, the different ligands (DA, BIX) were dissolved in deuterated methanol, acidified with deuterated hydrochloric acid, and characterized by ${ }^{1} \mathrm{H}$ NMR spectroscopy.

Simultaneous Determination of Monoamines Using HPLCECD. An HPLC-ECD method for the simultaneous quantification of monoamines (including DA, DOPAC, and HVA) was adapted from previously described methods. ${ }^{81}$ In brief, analyses were performed using a Chromolith Performance RP-18e $(100 \mathrm{~mm} \times 4.6 \mathrm{~mm}$, Merck-Millipore, Darmstadt, Germay) column in an Elite LaChrom system from Hitachi (Tokyo, Japan) coupled to a Coulochem 5100A electrochemical detector from ESA (Chelmsford, MA, USA). The detector was equipped with a 5011 dual-electrode analytical cell with porous graphite electrodes set at +5 and $+400 \mathrm{mV}$ (for electrodes 1 and 2 , respectively). Before the analysis, the column was preequilibrated in the mobile phase containing $99 \% \mathrm{v} / \mathrm{v}$ of a buffered aqueous solution $(0.1 \mathrm{M}$ citric acid, $0.05 \mathrm{mM}$ EDTA, $1.2 \mathrm{mM}$ sodium octyl sulfate) with $1 \%$ acetonitrile. The final $\mathrm{pH}$ of the solution was adjusted to $\mathrm{pH}=2.7$ with triethylamine. Elution of the monoamines was performed with an isocratic elution of the mobile phase, at $25{ }^{\circ} \mathrm{C}$ and a flow rate of $1 \mathrm{~mL} / \mathrm{min}$. Before analysis, samples of nanoparticles, culture medium, or cell lysates were centrifuged at $15000 \mathrm{~g}$ for $10 \mathrm{~min}$ at $4{ }^{\circ} \mathrm{C}$ and diluted in homogenization buffer $(0.25 \mathrm{M}$ perchloric acid, $100 \mu \mathrm{M}$ sodium bisulfite, $250 \mu \mathrm{M}$ EDTA), and $20 \mu \mathrm{L}$ of each sample was injected into the HPLC system. Calibration curves of DA, DOPAC, HVA, LDOPA, NA, DHBA, 5HIIA, 3-MT, and 5-HT were prepared by injecting different concentrations from 50 to $1000 \mathrm{pg} / \mathrm{mL}$. All samples and standards were prepared and analyzed in triplicate. The amount of each monoamine present in the samples was calculated from the corresponding calibration curve adjusted to a linear regression model (in all cases, with $R^{2}>0.99$ ). Instrument control, data acquisition, and monoamine determinations were carried out using EZChrom Elite version 3.1.7 software.

Cell Culture. The human BE(2)-M17 (ATCC CRL-2267) and HeLa (ATCC CCL-2) cell lines were maintained in Opti-MEM or minimum essential medium $\alpha$ (MEM- $\alpha)$ medium, respectively. Both media were further supplemented with $10 \%$ (v/v) FBS. Cells were grown under a highly humidified atmosphere of $95 \%$ air with $5 \%$ $\mathrm{CO}_{2}$ at $37^{\circ} \mathrm{C}$.

In Vitro Cytotoxicity Experiments. The cytotoxicity of the free ligands (DA, BIX, and iron acetate) and DA-NCPs toward BE(2)M17 and HeLa cells was evaluated by a resazurin-based assay using the PrestoBlue cell viability reagent. Briefly, BE(2)-M17 cells were seeded in 96-well plates at a concentration of $3 \times 10^{3}$ cells per well and incubated for $24 \mathrm{~h}$. After incubation, cells were treated with DA, $\mathrm{BIX}$, iron acetate, and DA-NCPs at different concentrations (ranging from 0 to $100 \mu \mathrm{g} / \mathrm{mL}(\mathrm{w} / \mathrm{v})$ for BIX and iron acetate or from 0 to $250 \mu \mathrm{g} / \mathrm{mL}(\mathrm{w} / \mathrm{v})$ for DA and DA-NCP). After $48 \mathrm{~h}$ of incubation, aliquots of $10 \mu \mathrm{L}$ of the PrestoBlue cell viability reagent solution were added to each well and cells were incubated for $1 \mathrm{~h}$ at $37^{\circ} \mathrm{C}$ in the absence of light and in the presence of a highly humidified atmosphere of $95 \%$ air with $5 \% \mathrm{CO}_{2}$. Fluorescence $(\mathrm{ex} / \mathrm{em}=531 /$ $572 \mathrm{~nm}$ ) of each well was measured using a fluorescence microplate reader (PerkinElmer Victor 3V). Cell cytotoxicity was evaluated in terms of BE(2)-M17 cell growth inhibition in treated samples and expressed as percent of the control assay. All cytotoxicity experiments were performed at least in triplicate.

Reactive Oxygen Species Determination. For ROS determination, BE(2)-M17 cells were seeded in a 96-well plate at a density of $10 \times 10^{4}$ cells/well. After $24 \mathrm{~h}$, DCFDA was added at a concentration of $25 \mu \mathrm{M}$, and the cells were incubated at $37{ }^{\circ} \mathrm{C}(5 \%$ $\mathrm{CO}_{2}$ ), in the absence of light, for $30 \mathrm{~min}$. DCFDA is a nonfluorescent and permeable dye that, after cleavage and oxidation by ROS or intracellular esterases, generates dichlorofluorescein (DCF), a fluorescent nonpermeable compound. All conditions were tested in the presence or absence of a natural antioxidant, AA, which, additionally, would avoid DA autoxidation. After incubation, the media was replaced, and cells were exposed for 24 $\mathrm{h}$ to DA, DA-NCPs (with an equivalent DA concentration of 100, 10, and $1 \mu \mathrm{g} / \mathrm{mL}$ ), and iron acetate (at concentrations corresponding to the same as the iron present in DA-NCPs). Hydrogen peroxide $\left(\mathrm{H}_{2} \mathrm{O}_{2}\right)$ was used as a positive control at a concentration of $250 \mu \mathrm{M}$. Cell culture medium was supplemented or not with AA at $20 \mu \mathrm{g} / \mathrm{mL}$. The fluorescence of each well was measured at $535 \mathrm{~nm}$ with a Victor3 microplate reader (PerkinElmer), after excitation at $485 \mathrm{~nm}$. Results are reported as an average of two independent experiments. The analysis of variance was evaluated with one-way ANOVA performed for ROS assays. The comparison between different groups was assessed by applying the Tukey-HSD test. Significance level was set to 0.05 .

Cellular Uptake of DA and DA-NCPs and Metabolism Experiments. To study the uptake and metabolism of free DA and DA-NCPs, human DArgic neuroblastoma BE(2)-M17 cells were seeded at a density of $2.5 \times 10^{5}$ cells/plate onto $60 \mathrm{~mm}$ culture plates and grown for $24 \mathrm{~h}$ (to reach about $60-70 \%$ confluence). After incubation, the medium was removed, and cells were treated with a control medium or with a medium containing the specified concentrations of free DA or DA-NCPs for 2, 6, and $24 \mathrm{~h}$. To prevent $\mathrm{DA}$ oxidation during treatments, media were further supplemented with $20 \mu \mathrm{g} / \mathrm{mL}$ AA.

For DA quantification, immediately before cell recovery, aliquots of $90 \mu \mathrm{L}$ of each culture medium supernatant were collected, mixed with $10 \mu \mathrm{L}$ of a $10 \times$ homogenization solution containing $2.5 \mathrm{M}$ perchloric acid, $1 \mathrm{mM}$ sodium bisulfite, and $2.5 \mathrm{mM}$ EDTA, and sonicated for $10 \mathrm{~s}$ on ice. For intracellular monoamine determinations, treated cells were washed three times with cold PBS, scraped, and centrifuged at $1000 \mathrm{~g}$ for $5 \mathrm{~min}$. The resultant pellets were resuspended in a $1 \times$ homogenization solution containing 0.25 $\mathrm{M}$ perchloric acid, $100 \mu \mathrm{M}$ sodium bisulfite, and $250 \mu \mathrm{M}$ EDTA, and samples were sonicated for $15 \mathrm{~s}$ on ice. The samples were centrifuged for $40 \mathrm{~min}$ at $4{ }^{\circ} \mathrm{C}$. The pellets were dispersed in PBS, and the total 
amount of protein was determined by the BCA or Bradford protein assay kits (Thermo Fisher Scientific, Waltham, MA, USA). All samples were kept at $-20{ }^{\circ} \mathrm{C}$ until HPLC-ECD analysis. Uptake rates were determined by measuring the intracellular levels of DA over time. In the case of DA, data were fitted to the following MichaelisMenten equation: $\frac{\mathrm{d}[\mathrm{DA}]}{\mathrm{d} t}=\frac{V_{\max }}{\frac{K_{m}}{[\mathrm{DA}]}+1}$, where $V_{\max }$ is the maximal velocity as a function of the density of the transporters and $K_{\mathrm{m}}$ is the value of $[\mathrm{DA}]_{0}$ in which the rate of uptake is half of the $V_{\max }$.

Preclinical Evaluation. Animal studies were approved by the corresponding local ethics committees, according to the regional and state legislation (protocol CEA-OH-9685/CEEAH-3665). The animals used for the tolerability and MRI experiments were C57BL/6J female mice of 12 weeks of age, weighing $21.5 \pm 0.4 \mathrm{~g}$ $(n=9)$. These animals were purchased from Charles River Laboratories (l'Abresle, France) and housed in the animal facility of the Universitat Autònoma de Barcelona (UAB). MRI studies were performed at the joint nuclear magnetic resonance facility of the $\mathrm{UAB}$ and Centro de Investigación Biomédica en Red Bioingenieria, Biomateriales y Nanomedicina (CIBER-BBN) (Cerdanyola del Vallès, Spain), Unit 25 of NANBIOSIS, www.nanbiosis.es. The 7T Bruker BioSpec 70/30 USR spectrometer (Bruker BioSpin $\mathrm{GmbH}$, Ettlingen, Germany) equipped with a mini-imaging gradient set $(400 \mathrm{mT} / \mathrm{m})$ was used for magnetic resonance acquisitions. The in vivo biodistribution and behavioral experiments with SpragueDawley rats were performed at the laboratory of animal service within the Vall d'Hebron Research Institute (VHIR).

Tolerability of Systematically Administered DA-NCPS. Tolerability experiments were done following a previously reported protocol. ${ }^{82}$ In a typical experiment, increasing amounts of DA-NCPs (0.003-0.1 mmol Fe kg ${ }^{-1}$ ) were intravenously (i.v.) administered (via tail vein) to $n=3$ mice with a 2 -day rest period between injections. An additional group of $n=3$ wild-type (wt) mice was injected with the vehicle as control (PBS with $0.5 \mathrm{mM}$ mouse serum albumin (MSA)). Mice body weight and other welfare parameters were followed for 30 days after the last administration. In these conditions, a dose of $0.1 \mathrm{mmol} \mathrm{kg}{ }^{-1}$ of DA-NCPs proved safe, and no significant weight loss or toxicity symptoms were observed during injections and for the 30-day period after the last administration. This value perfectly matches safety administration ranges for related systems such as Fe-based nanoparticles. ${ }^{83,84}$

Intracerebroventricular Administration of the DA-NCPs. The in vivo distribution of the nanoparticles in the nigrostriatal system was assessed in Sprague-Dawley male rats (275-300 g) after intracerebroventricular administration. A permanent cannula (C313G/SPC $3.5 \mathrm{~mm}$, Plastics One) was placed in the right lateral ventricle (AP: -0.8 ; $\mathrm{L}:-1.6$; $\mathrm{DV}:-3.6)$ of the rats under isoflurane anesthesia using a Kopf stereotaxic frame. A dummy cannula was placed in the guide cannula to prevent the entry of material. Three weeks later, DA-NCPs (a total dose equivalent to $50 \mu \mathrm{g}$ of free DA) were infused into the lateral ventricle with an internal cannula with tubing attached to a $2.5 \mathrm{~mL}$ Hamilton syringe placed in a peristaltic pump (KDS 220). A $20 \mu \mathrm{L}$ amount of a DA-NCP suspension was infused at a $1 \mu \mathrm{L} / \mathrm{min}$ rate. The dummy cannula was placed back into the guide cannula after the administration. Rats were euthanized at 2, 6, and $24 \mathrm{~h}$ after the i.c.v. administration of the nanoparticles. The brains were removed, and both right and left ventral midbrains and striata were dissected and kept separately at $-80{ }^{\circ} \mathrm{C}$ until analysis.

In Vitro, ex Vivo, and in Vivo MRI Studies with DA-NCPs. For the in vitro quantitative magnetic resonance imaging phantoms (MRI-Phantoms), a $7.2 \mathrm{~cm}$ inner diameter volume coil was used for excitation and reception of NMR signals. The samples of DA-NCPs were dispersed using agarose $(1 \%)$ in $\mathrm{PBS}$ at $\mathrm{pH}=7.4$. Five samples at decreasing concentrations $(0.6,0.3,0.1,0.05$, and $0 \mathrm{mM}$ of metal $\mathrm{mL}^{-1}$ ) were prepared in $1.5 \mathrm{~mL}$ Eppendorf tubes. The total volume was adjusted up to $1 \mathrm{~mL}$ with agarose in PBS. The suspensions were dispersed in an ultrasound bath just before the solidification of medium and acquisition sequences for assessing longitudinal relaxation $\left(T_{1}\right)$. For $T_{1}$ maps, a rapid acquisition with relaxation enhancement (RARE) with variable repetition time (VTR) sequence was used. Echo time (TE) was $7.5 \mathrm{~ms}$ acquiring at 18 different repetition times (TR) ranging from 30 to $5000 \mathrm{~ms}$; field of view (FOV), $90 \times 60 \mathrm{~mm}$; matrix size (MTX), $128 \times 128(700 \mu \mathrm{m} \times 470$ $\mu \mathrm{m} /$ pixel); number of averages (NA), 1; RARE factor 2; and total acquisition time (TAT) $21 \mathrm{~min} 22 \mathrm{~s}$. Parameters for $T_{2}$ maps were multislice multiecho sequence (MSME); TR, $3 \mathrm{~s}$; TE ranged from 10 to $600 \mathrm{~ms}$ with $10 \mathrm{~ms}$ echo spacing; NA, 1; same geometry parameters as in $T_{1}$ maps; and TAT, 6 min $24 \mathrm{~s}$.

For the ex vivo post mortem MRI studies, a $72 \mathrm{~mm}$ inner diameter volume coil was used in combination with a dedicated mouse brain surface receiver coil. The DA-NCPs were dispersed in $2 \mathrm{~mL}$ of PBS added with $0.5 \mathrm{mM}$ BSA. The amount finally used for each animal was $5 \mathrm{nmol}$ of metal dissolved in $4 \mu \mathrm{L}$ of PBS-BSA solution $(n=3)$. The commercial solution of CA-based in GdDTPA $(n=3)$ was used as a standard to compare the enhancement obtained with the NCPs synthesized, and the vehicle was also injected for comparison purposes $(n=3)$. Three injection points were used in each mouse. All administrations were performed post mortem using a stereotactic holder, as already described. ${ }^{76}$ High-resolution $T_{1 \mathrm{w}}$ and $T_{2 \mathrm{w}} \mathrm{MR}$ images were obtained for each mouse for morphological characterization of the investigated tissue. $\mathrm{T}_{2 \mathrm{w}}$ images $\left(\mathrm{TR} / \mathrm{TE}_{\text {eff }}=4200 /-36\right.$ $\mathrm{ms}$ ) were acquired using a rapid acquisition with relaxation enhancement (RARE) sequence. Parameters for $\mathrm{T}_{2 \mathrm{w}}$ images were RARE factor $=8$; FOV, $19.2 \times 19.2 \mathrm{~mm}$; MTX, $256 \times 256(75 \mu \mathrm{m}$ $\times 75 \mu \mathrm{m} /$ pixel); NA, 4; TAT, $6 \min 43 \mathrm{~s}$. After this, a $T_{1 \mathrm{w}}$ image (TR/TE $350 / 10 \mathrm{~ms}$ ) was acquired with an MSME sequence with the same FOV and MTX; NA, 8; TAT, $11 \mathrm{~min} 56 \mathrm{~s}$. Three ROIs corresponding to the injection coordinates were manually defined after visual inspection in both the area of maximum enhancement and equivalent area of contralateral parenchyma using Paravision 5.1. The RCE-injection site ROI vs contralateral parenchymaobtained in each case was assessed. Only the slice with a better defined contrast-enhanced region was measured. For dual enhancement images (Figure $6 \mathrm{c}$ ), $T_{1 \mathrm{w}}$ and $T_{2 \mathrm{w}}$ images were processed with the algebraic algorithm ( $\mathrm{MR}$ signal $T_{1} / \mathrm{MR}$ signal $T_{2}$ ) provided by Paravision 5.1.

To examine the local distribution of DA-NCPs following intranasal administration, in vivo MRI was performed using a transmit volume resonator (72 $\mathrm{mm}$ inner diameter) for excitation and a dedicated rat brain surface coil as a receiver. Sprague-Dawley male rats (275-300 g) were anesthetized with isoflurane $1-2 \%$ in $\mathrm{O}_{2}$ at $1 \mathrm{~L} / \mathrm{min}$. Breathing $(60-80$ breaths $/ \mathrm{min})$ and body temperature $\left(37-38{ }^{\circ} \mathrm{C}\right)$ were constantly monitored (SA Instruments, Inc., New York, USA) during the MRI experiments. Rats were placed into a holder with bite-bar and ear-bars for optimal head immobilization. A series of $T_{1 \mathrm{w}}$ coronal images and $T_{1}$ mapping in the axial plane, covering the nasal cavity from the external naris to the olfactory bulb, were acquired before and immediately after intranasal administration of the nanoparticles. Following the initial MRI scanning, DA-NCPs (40 $\mu \mathrm{L}$, with a total dose equivalent to $200 \mu \mathrm{g}$ of free DA dispersed in PBS-MSA) were applied to the right nostril of the animal placed in a supine position by slow administration of drops within $10 \mathrm{~min}$. Immediately after DA-NCP administration, a second MRI scanning was carried out under identical experimental conditions. $T_{1 \mathrm{w}}$ images from seven coronal $1.5 \mathrm{~mm}$ slices were acquired with an MSME sequence with TR, $300 \mathrm{~ms}$; TE, $10 \mathrm{~ms}$; FOV, $40 \times 35 \mathrm{~mm}$; MTX, $256 \times 256(156 \mu \mathrm{m} \times 137 \mu \mathrm{m} /$ pixel $) ; \mathrm{NA}, 4$; and TAT, $3 \mathrm{~min} 50 \mathrm{~s}$. $T_{1}$ maps in the axial plane were obtained using a RAREVTR sequence with $7 \mathrm{~ms}$ TE and acquisition at 10 different TR values $(450,525,650,750,950,1200,1500,1900,2500$, and $5000 \mathrm{~ms})$. A total of 24 contiguous axial $1 \mathrm{~mm}$ slices were acquired with FOV, 35 $\times 35 \mathrm{~mm}$; MTX, $128 \times 128(273 \mu \mathrm{m} \times 273 \mu \mathrm{m} /$ pixel $)$; RARE factor, 1; and TAT, $32 \mathrm{~min} 54 \mathrm{~s}$. Data were fitted to an exponential curve using the ISA tool in ParaVision 5.1 in order to estimate $T_{1}$ values, as described in the Supporting Information. Mean $T_{1}$ values pre- and postadministration of DA-NCPs were obtained from ROIs of three consecutive sections. ROIs were manually defined in areas along the right nasal conduit showing high enhancement on $T_{1 \mathrm{w}}$ 
images postadministration, and afterward, the equivalent region from the preadministration data set was selected.

6-HyroxyDA-Lesioned Rat Model of PD. The 6-OHDA rat model of PD was prepared as described previously. ${ }^{85}$ In brief, for stereotaxic surgery, Sprague-Dawley male rats $(250-275 \mathrm{~g})$ were anesthetized with isoflurane $\left(0.5-1.5 \%\right.$ in $\left.\mathrm{O}_{2}\right)$. The animals were positioned on a stereotaxic frame (Kopf Instruments, Tujunga, CA, USA), and 6-OHDA was unilaterally injected in the right hemisphere of the brain (2 $\mu \mathrm{L}$ of freshly dissolved 6-OHDA hydrochloride prepared with $0.02 \%$ ascorbate, Sigma-Aldrich, St. Louis, MO, USA) using a 33-G needle Hamilton syringe (Hamilton Bonaduz AG., Bonaduz, Switzerland). The injection of the neurotoxin was performed into the medial forebrain bundle, with the needle placed $4.0 \mathrm{~mm}$ anterior to the interaural line, $1.3 \mathrm{~mm}$ lateral to the midline, and $8.4 \mathrm{~mm}$ ventral to the surface of the skull, according to the reference coordinates from the Paxinos and Watson rat brain atlas. ${ }^{86}$ After stereotaxic injection, the animals were placed in their cages and monitored until recovery.

Intranasal Administration of DA-NCPs. For the pharmacokinetic study, 18 healthy Sprague-Dawley rats $(275-300 \mathrm{~g})$ were randomly divided into two experimental groups and intranasally administered with vehicle (PBS-MSA solution) or with DA-NCPs dispersed in PBS with $0.25 \mathrm{mM}$ MSA. To perform the intranasal administrations, the animals were anesthetized with isoflurane (0.5$1.5 \%$ in $\left.\mathrm{O}_{2}\right)$ and put in a supine position. The vehicle $(20 \mu \mathrm{L})$ or DA-NCPs $(20 \mu \mathrm{L}$, with a total dose equivalent to $50 \mu \mathrm{g}$ of free DA) were slowly applied to the right and left nostrils using an Eppendorf pipet. The animals were euthanized at 30 and $120 \mathrm{~min}$ after the intranasal administration of the nanoparticles. Then, the brains were removed, and both right and left ventral midbrains and striata were dissected and kept separately at $-80{ }^{\circ} \mathrm{C}$ until HPLC analysis. To evaluate the performance of DA-NCP administration in comparison with free DA, an additional experiment was performed. In this experiment, nine healthy Sprague-Dawley rats (275-300 g) were randomly divided into three groups and intranasally administered with either vehicle, DA-NCPs, or free DA. These animals were sacrificed $120 \mathrm{~min}$ administration postadministration, and the brains dissected as above. The levels of DA present in the samples were determined by HPLC-ECD as described hereafter.

For behavioral testing, 18 6-OHDA-lesioned Sprague-Dawley rats were divided into three treatment groups. The animals were first allowed to habituate to the room and manipulation for at least 1 week before treatments. Two months after the surgery, one group of 6-OHDA-lesioned rats received a semichronic intranasal administration of DA-NCPs at doses equivalent to $800 \mu \mathrm{g}$ of free DA (four daily doses of $50 \mu \mathrm{g}$, between 8 a.m. and 15 p.m.) per day, for 4 days. A second group of PD rats received equivalent doses of free DA. The third group of animals was treated with vehicle (PBS-MSA solution), as a control condition. After semichronic drug administration, rats were subjected to behavioral analyses.

Apomorphine-Induced Rotation Test. Rats with unilateral 6OHDA lesions of the nigrostriatal pathway exhibit contralateral rotational behavior when administered with DA agonists such as apomorphine. ${ }^{85}$ This phenomenon can be explained by the hypersensitization of the injured striatum. For assessment of circling behavior, the 6-OHDA-lesioned rats were challenged with $0.5 \mathrm{mg} / \mathrm{kg}$ of apomorphine (Sigma-Aldrich, St. Louis, MO, USA) 6 weeks after striatal 6-OHDA injections and after semichronic administration of the nanoparticles. Note that the first evaluation was carried out to determine the effect of the unilateral 6-OHDA lesion on each animal. To perform the rotation tests, rats were introduced inside individual circular cages and connected to an automated rotometer (LE 902 rotameter, Harvard Apparatus, Holliston, MA, USA). The animals were then habituated to the environment for $15 \mathrm{~min}$. Afterward, a subcutaneous injection of apomorphine was given to each of the animals, and the rotational activity was recorded for $1 \mathrm{~h}$. The rotational activity was determined by calculating the total number of net contralateral complete $\left(360^{\circ}\right)$ rotations after subtraction of the contralateral turns. The total duration of the rotational behavior after treatment was also determined for each of the animals and compared with the duration of the rotational behavior before the treatment.

\section{ASSOCIATED CONTENT}

\section{SI Supporting Information}

The Supporting Information is available free of charge at https://pubs.acs.org/doi/10.1021/acsnano.1c00453.

Details including Materials and Methods, HPLC quantification of bix in the DA-NCPs, DA content of DA-NCPs determined by HPLC, ICP-MS quantification of iron in DA-NCPs, in vitro release kinetics under physiological conditions and processing, postprocessing of MR data and encapsulation of EGF; additional tables (Table S1-S4) and figures (Figure S1-S18) (PDF)

\section{AUTHOR INFORMATION}

\section{Corresponding Authors}

Julia Lorenzo - Institut de Biotecnologia $i$ de Biomedicina (IBB), Universitat Autònoma de Barcelona, 08193 Bellaterra, Barcelona, Spain; Departament de Bioquímica $i$ Biologia Molecular, Unitat de Bioquímica de Biociencies, Edifici C, Universitat Autònoma de Barcelona, 08193 Cerdanyola del Valles, Spain; Email: julia.lorenzo@uab.cat Daniel Ruiz-Molina - Catalan Institute of Nanoscience and Nanotechnology (ICN2), CSIC and BIST, 08193 Bellaterra, Barcelona, Spain; 이이이.org/0000-0002-6844-8421; Email: dani.ruiz@icn2.cat

\section{Authors}

Javier García-Pardo - Catalan Institute of Nanoscience and Nanotechnology (ICN2), CSIC and BIST, 08193 Bellaterra, Barcelona, Spain; Institut de Biotecnologia $i$ de Biomedicina (IBB), Universitat Autònoma de Barcelona, 08193 Bellaterra, Barcelona, Spain; Departament de Bioquímica $i$ Biologia Molecular, Unitat de Bioquímica de Biociencies, Edifici C, Universitat Autònoma de Barcelona, 08193 Cerdanyola del Valles, Spain

Fernando Novio - Catalan Institute of Nanoscience and Nanotechnology (ICN2), CSIC and BIST, 08193 Bellaterra, Barcelona, Spain; Departament de Química, Universitat Autònoma de Barcelona (UAB), 08193 Cerdanyola del Valles, Barcelona, Spain; ○ orcid.org/0000-0002-15173612

Fabiana Nador - Catalan Institute of Nanoscience and Nanotechnology (ICN2), CSIC and BIST, 08193 Bellaterra, Barcelona, Spain

Ivana Cavaliere - Catalan Institute of Nanoscience and Nanotechnology (ICN2), CSIC and BIST, 08193 Bellaterra, Barcelona, Spain

Salvio Suárez-García - Catalan Institute of Nanoscience and Nanotechnology (ICN2), CSIC and BIST, 08193 Bellaterra, Barcelona, Spain; 아이. orcid.org/0000-0002-4156-0579

Silvia Lope-Piedrafita - Centro de Investigacion Biomédica en Red en Bioingeniería, Biomateriales y Nanomedicina (CIBER-BBN), 08193 Cerdanyola del Vallés, Spain; Servei de Ressonància Magnètica Nuclear, Universitat Autònoma de Barcelona, 08193 Cerdanyola del Valles, Spain

Ana Paula Candiota - Institut de Biotecnologia $i$ de Biomedicina (IBB), Universitat Autònoma de Barcelona, 08193 Bellaterra, Barcelona, Spain; Departament de Bioquímica i Biologia Molecular, Unitat de Bioquímica de Biociencies, Edifici C, Universitat Autònoma de Barcelona, 08193 Cerdanyola del Valles, Spain; Centro de Investigacion 
Biomédica en Red en Bioingeniería, Biomateriales y Nanomedicina (CIBER-BBN), 08193 Cerdanyola del Vallés, Spain

Jordi Romero-Gimenez - Neurodegenerative Diseases Research Group, Vall d'Hebron Research Institute (VHIR)Center for Networked Biomedical Research on Neurodegenerative Diseases (CIBERNED), Edifici Collserola Hospital Universitari Vall d'Hebron, 08035 Barcelona, Spain

Beatriz Rodríguez-Galván - Neurodegenerative Diseases Research Group, Vall d'Hebron Research Institute (VHIR)Center for Networked Biomedical Research on Neurodegenerative Diseases (CIBERNED), Edifici Collserola Hospital Universitari Vall d'Hebron, 08035 Barcelona, Spain

Jordi Bové - Neurodegenerative Diseases Research Group, Vall d'Hebron Research Institute (VHIR)-Center for Networked Biomedical Research on Neurodegenerative Diseases (CIBERNED), Edifici Collserola Hospital Universitari Vall d'Hebron, 08035 Barcelona, Spain

Miquel Vila - Neurodegenerative Diseases Research Group, Vall d'Hebron Research Institute (VHIR)-Center for Networked Biomedical Research on Neurodegenerative Diseases (CIBERNED), Edifici Collserola Hospital Universitari Vall d'Hebron, 08035 Barcelona, Spain; Institut de Neurociencies, Departament de Bioquímica i Biologia Molecular, Universitat Autònoma de Barcelona, 08193 Cerdanyola del Valles, Spain; ICREA-Institució Catalana de Recerca i Estudis Avancats, 08010 Barcelona, Spain; (1) orcid.org/0000-0002-1352-989X

Complete contact information is available at:

https://pubs.acs.org/10.1021/acsnano.1c00453

\section{Author Contributions}

J.G.-P., M.V., J.B., J.L., and D.R.-M. designed the conceptual framework of the study and experiments. F.N. designed, performed, and analyzed experiments for the initial synthesis and characterization of the DA-NCPs. J.G.-P., I.C., and F.N. synthesized DA-NCPs and contributed to their structural and chemical characterization. J.G.-P. performed the DA-NCP uptake and metabolism experiments. J.G.-P. and J.L. performed cytotoxicity experiments. I.C. and J.L. contributed to ROS measurements. S.S.-G., S.L.-P., and A.P.C. performed tolerability experiments and MRI determinations. J.G.-P., J.R.G., B.R.-G., and. J.B. contributed to i.c.v. administration of the nanoparticles and DA-NCP biodistribution experiments. J.G.P., B.R.-G., and. J.B. performed the 6-OHDA lesions, the intranasal administrations, and behavioral tests. J.G.-P. and F.N. performed HPLC-ECD determinations. J.G.-P. and D.R.M. wrote the manuscript with contributions and valuable comments from all authors.

\section{Notes}

The authors declare no competing financial interest.

\section{ACKNOWLEDGMENTS}

This work was supported by grants RTI2018-098027-B-C21 and RTI2018-098027-B-C22 from the Spanish Government funds and by the European Regional Development Fund (ERDF). The ICN2 is funded by the CERCA program/ Generalitat de Catalunya. The ICN2 is supported by the Severo Ochoa Centres of Excellence program, funded by the Spanish Research Agency (AEI, grant no. SEV-2017-0706).
M.V. received funding from "la Caixa" Foundation (ID 1178 100010434 , under the agreement LCF/PR/HR17/52150003) 1179, Ministry of Economy and Competitiveness (MINECO, Spain) (SAF2016-77541-R) and The Michael J. Fox Foundation for Parkinson's Research 1180 (Grant ID: 15291.01). A.P.C. received funding from the ATTRACT project funded by the EC under Grant Agreement 777222. The authors thank the support from COST Action CA17121. We thank Susana Benítez Andreu and the Department of Biochemistry and Molecular Biology at the Universitat Autonoma de Barcelona for the exceptional technical support and assistance with HPLC-ECD determinations and data analysis. Special thanks to Karl-Richard Reutter (instagram: NIR.io_art) and Javier Saiz from 3D Science Visuals for contributing to graphic design and scientific illustrations. We also thank Prof. Azzedine Bousseksou and Dr. Gábor Molnár for Mössbauer experiments.

\section{REFERENCES}

(1) Kalia, L. V.; Lang, A. E. Parkinson's Disease. Lancet 2015, 386 (9996), 896-912.

(2) Pringsheim, T.; Jette, N.; Frolkis, A.; Steeves, T. D. The Prevalence of Parkinson's Disease: a Systematic Review and MetaAnalysis. Mov. Disord. 2014, 29 (13), 1583-90.

(3) Dauer, W.; Przedborski, S. Parkinson's Disease: Mechanisms and Models. Neuron 2003, 39 (6), 889-909.

(4) Gibb, W. R.; Lees, A. J. Anatomy, Pigmentation, Ventral and Dorsal Subpopulations of the Substantia Nigra, and Differential Cell Death in Parkinson's Disease. J. Neurol., Neurosurg. Psychiatry 1991, 54 (5), 388-96.

(5) Gibb, W. R.; Lees, A. J. The Significance of the Lewy Body in the Diagnosis of Idiopathic Parkinson's Disease. Neuropathol. Appl. Neurobiol. 1989, 15 (1), 27-44.

(6) Bernheimer, H.; Birkmayer, W.; Hornykiewicz, O.; Jellinger, K.; Seitelberger, F. Brain Dopamine and the Syndromes of Parkinson and Huntington. Clinical, Morphological and Neurochemical Correlations. J. Neurol. Sci. 1973, 20 (4), 415-55.

(7) Hirsch, E.; Graybiel, A. M.; Agid, Y. A. Melanized Dopaminergic Neurons are Differentially Susceptible to Degeneration in Parkinson's Disease. Nature 1988, 334 (6180), 345-8.

(8) Connolly, B. S.; Lang, A. E. Pharmacological Treatment of Parkinson Disease: a Review. Jama 2014, 311 (16), 1670-83.

(9) Marsden, C. D.; Parkes, J. D. “On-Off” Effects in Patients with Parkinson's Disease on Chronic Levodopa Therapy. Lancet 1976, 1 (7954), 292-6.

(10) Obeso, J. A.; Grandas, F.; Vaamonde, J.; Luquin, M. R.; Artieda, J.; Lera, G.; Rodriguez, M. E.; Martinezlage, J. M. Motor Complications Associated with Chronic Levodopa Therapy in Parkinsons-Disease. Neurology 1989, 39 (11), 11-19.

(11) van Wamelen, D. J.; Grigoriou, S.; Chaudhuri, K. R.; Odin, P. Continuous Drug Delivery Aiming Continuous Dopaminergic Stimulation in Parkinson's Disease. J. Parkinson's Dis. 2018, 8 (s1), S65-S72.

(12) Trapani, A.; Mandracchia, D.; Tripodo, G.; Cometa, S.; Cellamare, S.; De Giglio, E.; Klepetsanis, P.; Antimisiaris, S. G. Protection of Dopamine Towards Autoxidation Reaction by Encapsulation into Non-Coated- or Chitosan- or Thiolated Chitosan-Coated-Liposomes. Colloids Surf., B 2018, 170, 11-19.

(13) Lopalco, A.; Cutrignelli, A.; Denora, N.; Lopedota, A.; Franco, M.; Laquintana, V. Transferrin Functionalized Liposomes Loading Dopamine HCl: Development and Permeability Studies Across an in Vitro Model of Human Blood-Brain Barrier. Nanomaterials 2018, 8 (3), 178.

(14) Fabregat, G.; Gimenez, A.; Diaz, A.; Puiggali, J.; Aleman, C. Dual-Functionalization Device for Therapy through Dopamine Release and Monitoring. Macromol. Biosci. 2018, 18 (5), No. e1800014. 
(15) Ren, Y.; Zhao, X.; Liang, X.; Ma, P. X.; Guo, B. Injectable Hydrogel Based on Quaternized Chitosan, Gelatin and Dopamine as Localized Drug Delivery System to Treat Parkinson's Disease. Int. J. Biol. Macromol. 2017, 105, 1079-1087.

(16) Ragusa, A.; Giudetti, A. M.; Ciccarela, G.; Gaballo, A. Neuroprotective Investigation of Chitosan Nanoparticles for Dopamine Delivery. Appl. Sci. 2018, 8 (4), 474.

(17) Trapani, A.; De Giglio, E.; Cafagna, D.; Denora, N.; Agrimi, G.; Cassano, T.; Gaetani, S.; Cuomo, V.; Trapani, G. Characterization and Evaluation of Chitosan Nanoparticles for Dopamine Brain Delivery. Int. J. Pharm. 2011, 419 (1-2), 296-307.

(18) Guo, Q.; Yang, X.; Lin, B.; Zhu, Z.; Lu, Z.; Li, X.; Zhao, Y.; Mao, L.; Shen, S.; Cheng, H.; Zhang, J.; Deng, L.; Fan, J.; Xi, Z.; Li, R.; Li, C. M. Functional Single-Walled Carbon Nanotubes 'CAR' for Targeting Dopamine Delivery into the Brain of Parkinsonian Mice. Nanoscale 2017, 9, 10832-10845.

(19) Kondaveeti, S.; Petri, D. F. Alginate/Magnetite Hybrid Beads for Magnetically Stimulate Release. Colloids Surf., B 2016, 138, 94101.

(20) Khan, M. S.; Pandey, S.; Talib, A.; Bhaisare, M. L.; Wu, H. F. Controlled Delivery of Dopamine Hydrochloride Using Surface Modified Carbon Dots for Neuro Diseases. Colloids Surf., B 2015, $134,140-6$.

(21) Aguilera, G.; West, R. M.; Gonzalez-Monterrubio, E.; AnguloMolina, A.; Arias-Carrión, Ó.; Méndez-Rojas, M. A. Carboxymethyl Cellulose Coated Magnetic Nanoparticles Transport Across a Human Lung Microvascular Endothelial Cell Model of the Blood-Brain Barrier. Nanoscale Adv. 2019, 1, 671-685.

(22) Tang, S.; Wang, A.; Yan, X.; Chu, L.; Yang, X.; Song, Y.; Sun, K.; Yu, X.; Liu, R.; Wu, Z.; Xue, P. Brain-Targeted Intranasal Delivery of Dopamine with Borneol and Lactoferrin co-Modified Nanoparticles for Treating Parkinson's Disease. Drug Delivery 2019, 26 (1), 700-707.

(23) Rashed, E. R.; Abd El-Rehim, H. A.; El-Ghazaly, M. A. Potential Efficacy of Dopamine Loaded-PVP/PAA Nanogel in Experimental Models of Parkinsonism: Possible Disease Modifying Activity. J. Biomed. Mater. Res., Part A 2015, 103 (5), 1713-20.

(24) Pahuja, R.; Seth, K.; Shukla, A.; Shukla, R. K.; Bhatnagar, P.; Chauhan, L. K.; Saxena, P. N.; Arun, J.; Chaudhari, B. P.; Patel, D. K.; Singh, S. P.; Shukla, R.; Khanna, V. K.; Kumar, P.; Chaturvedi, R. K.; Gupta, K. C. Trans-Blood Brain Barrier Delivery of DopamineLoaded Nanoparticles Reverses Functional Deficits in Parkinsonian Rats. ACS Nano 2015, 9 (5), 4850-71.

(25) Schwarz, S. T.; Rittman, T.; Gontu, V.; Morgan, P. S.; Bajaj, N.; Auer, D. P. T1-Weighted MRI Shows Stage-Dependent Substantia Nigra Signal Loss in Parkinson's Disease. Mov. Disord. 2011, 26 (9), 1633-8.

(26) Zucca, F. A.; Segura-Aguilar, J.; Ferrari, E.; Munoz, P.; Paris, I.; Sulzer, D.; Sarna, T.; Casella, L.; Zecca, L. Interactions of Iron, Dopamine and Neuromelanin Pathways in Brain Aging and Parkinson's Disease. Prog. Neurobiol. 2017, 155, 96-119.

(27) Chen, A.; Sun, J.; Liu, S.; Li, L.; Peng, X.; Ma, L.; Zhang, R. The Effect of Metal Ions on Endogenous Melanin Nanoparticles Used as Magnetic Resonance Imaging Contrast Agents. Biomater. Sci. 2020, 8 (1), 379-390.

(28) Chen, L.; Liu, L.; Zhang, X.; Shi, W.; Ge, D.; Sun, Y. Fe2+/ Fe3+ Ions Chelated with Ultrasmall Polydopamine Nanoparticles Inducing Ferroptosis for Cancer Therapy. ACS Biomater. Sci. Eng. 2019, 5, 4861-4869.

(29) Xia, L. C.; Zhu, H.; Yang, Z. Radionuclides Doped DopamineMelanin Nanoparticle as an Efficient Imaging Probe for in Vivo PET Imaging. J. Nucl. Med. 2019, 60, 1017.

(30) Liu, H.; Liu, Y.; Pang, X.; Wu, Y.; Zhou, Z.; Zhang, P.; Zhang, W.; Liu, G.; Chen, X. Novel Intrapolymerization Doped ManganeseEumelanin Coordination Nanocomposites with Ultrahigh Relaxivity and Their Application in Tumor Theranostics. Adv. Sci. 2018, 5, 1800032.

(31) Ang, J. M.; Du, Y.; Tay, B. Y.; Zhao, C.; Kong, J.; Stubbs, L. P.; Lu, X. One-Pot Synthesis of Fe(III)-Polydopamine Complex
Nanospheres: Morphological Evolution, Mechanism, and Application of the Carbonized Hybrid Nanospheres in Catalysis and Zn-Air Battery. Langmuir 2016, 32 (36), 9265-75.

(32) Yang, L.; Kong, J.; Zhou, D.; Ang, J. M.; Phua, S. L.; Yee, W. A.; Liu, H.; Huang, Y.; Lu, X. Transition-Metal-Ion-Mediated Polymerization of Dopamine: Mussel-Inspired Approach for the Facile Synthesis of Robust Transition-Metal Nanoparticle-Graphene Hybrids. Chem. - Eur. J. 2014, 20 (25), 7776-83.

(33) Son, H. Y.; Lee, H.; Nam, Y. S. Silver-Polydopamine Hybrid Coatings of Electrospun Poly(vinyl alcohol) Nanofibers. Macromol. Mater. Eng. 2013, 289, 547-554.

(34) Zhang, L.; Jian, R.-K.; Wang, D.-Y. Bioinspired Iron-Loaded Polydopamine Nanospheres as Green Flame Retardant for Epoxy Resin via Free Radical Scavenging and Catalytic Charring. J. Mater. Chem. A 2020, 8 (5), 2529-2538.

(35) Zhu, Y.; Xin, N.; Qiao, Z.; Chen, S.; Zeng, L.; Zhang, Y.; Wei, D.; Sun, J.; Fan, H. Novel Tumor-Microenvironment-Based Sequential Catalytic Therapy by Fe(II)-Engineered Polydopamine Nanoparticles. ACS Appl. Mater. Interfaces 2019, 11 (46), 4301843030 .

(36) Li, Y.; Xie, Y.; Wang, Z.; Zang, N.; Carniato, F.; Huang, Y.; Andolina, C. M.; Parent, L. R.; Ditri, T. B.; Walter, E. D.; Botta, M.; Rinehart, J. D.; Gianneschi, N. C. Structure and Function of IronLoaded Synthetic Melanin. ACS Nano 2016, 10 (11), 10186-10194.

(37) Longo, D. L.; Stefania, R.; Aime, S.; Oraevsky, A. MelaninBased Contrast Agents for Biomedical Optoacoustic Imaging and Theranostic Applications. Int. J. Mol. Sci. 2017, 18 (8), 1719.

(38) Lemaster, J. E.; Hariri, A.; Chen, F.; Hu, Z.; Huang, Y.; Barback, C. V.; Cochran, R.; Gianneschi, N. C.; Jokerst, J. V. Gadolinium Doping Enhances the Photoacoustic Signal of Synthetic Melanin Nanoparticles: A Dual Modality Contrast Agent for Stem Cell Imaging. Chem. Mater. 2011, 31, 251-259.

(39) Ju, K. Y.; Lee, Y.; Lee, S.; Park, S. B.; Lee, J. K. Bioinspired Polymerization of Dopamine to Generate Melanin-Like Nanoparticles Having an Excellent Free-Radical-Scavenging Property. Biomacromolecules 2011, 12 (3), 625-32.

(40) Miao, Z. H.; Wang, H.; Yang, H.; Li, Z. L.; Zhen, L.; Xu, C. Y. Intrinsically Mn2+-Chelated Polydopamine Nanoparticles for Simultaneous Magnetic Resonance Imaging and Photothermal Ablation of Cancer Cells. ACS Appl. Mater. Interfaces 2015, 7 (31), 16946-52.

(41) Novio, F.; Lorenzo, J.; Nador, F.; Wnuk, K.; Ruiz-Molina, D. Carboxyl Group $\left(-\mathrm{CO}_{2} \mathrm{H}\right)$ Functionalized Coordination Polymer Nanoparticles as Efficient Platforms for Drug Delivery. Chem. - Eur. J. 2014, 20 (47), 15443-50.

(42) Nador, F.; García-Pardo, J.; Lorenzo, J.; Solorzano, R.; RuizMolina, D.; Novio, F. Dual-Fluorescent Nanoscale Coordination Polymers via a Mixed-Ligand Synthetic Strategy and Their Use for Multichannel Imaging. ChemNanoMat 2018, 4, 183-193.

(43) Jo, D. H.; Chiou, Y. M.; Que, L., Jr. Models for Extradiol Cleaving Catechol Dioxygenases: Syntheses, Structures, and Reactivities of Iron(II)-Monoanionic Catecholate Complexes. Inorg. Chem. 2001, 40 (13), 3181-90.

(44) Solorzano, R.; Tort, O.; Garcia-Pardo, J.; Escriba, T.; Lorenzo, J.; Arnedo, M.; Ruiz-Molina, D.; Alibes, R.; Busque, F.; Novio, F. Versatile Iron-Catechol-Based Nanoscale Coordination Polymers with Antiretroviral Ligand Functionalization and their use as Efficient Carriers in HIV/AIDS Therapy. Biomater. Sci. 2019, 7 (1), 178-186.

(45) Amorin-Ferre, L.; Busque, F.; Bourdelande, J. L.; Ruiz-Molina, D.; Hernando, J.; Novio, F. Encapsulation and Release Mechanisms in Coordination Polymer Nanoparticles. Chem. - Eur. J. 2013, 19 (51), 17508-16.

(46) Aires, A.; Cabrera, D.; de la Cueva, L.; Salas, G.; Teran, F. J.; Cortajarena, A. L. BSA-Coated Magnetic Nanoparticles for Improved Therapeutic Properties. J. Mater. Chem. B 2015, 3, 6239-6247.

(47) Mariam, J.; Sivakami, S.; Dongre, P. M. Albumin Corona on Nanoparticles - a Strategic Approach in Drug Delivery. Drug Delivery 2016, 23 (8), 2668-2676.

(48) Moore, T. L.; Rodriguez-Lorenzo, L.; Hirsch, V.; Balog, S.; Urban, D.; Jud, C.; Rothen-Rutishauser, B.; Lattuada, M.; Petri-Fink, 
A. Nanoparticle Colloidal Stability in Cell Culture Media and Impact on Cellular Interactions. Chem. Soc. Rev. 2015, 44 (17), 6287-305. (49) Aggarwal, P.; Hall, J. B.; McLeland, C. B.; Dobrovolskaia, M. A.; McNeil, S. E. Nanoparticle Interaction with Plasma Proteins as it Relates to Particle Biodistribution, Biocompatibility and Therapeutic Efficacy. Adv. Drug Delivery Rev. 2009, 61 (6), 428-37.

(50) Holten-Andersen, N.; Harrington, M. J.; Birkedal, H.; Lee, B. P.; Messersmith, P. B.; Lee, K. Y.; Waite, J. H. pH-Induced MetalLigand Cross-Links Inspired by Mussel Yield Self-Healing Polymer Networks with Near-Covalent Elastic Moduli. Proc. Natl. Acad. Sci. U. S. A. 2011, 108 (7), 2651-5.

(51) Alberio, T.; Lopiano, L.; Fasano, M. Cellular Models to Investigate Biochemical Pathways in Parkinson's Disease. FEBS J. 2012, 279 (7), 1146-55.

(52) Zhang, S.; Wang, R.; Wang, G. Impact of Dopamine Oxidation on Dopaminergic Neurodegeneration. ACS Chem. Neurosci. 2019, 10 (2), 945-953.

(53) Kalyanaraman, B.; Darley-Usmar, V.; Davies, K. J.; Dennery, P. A.; Forman, H. J.; Grisham, M. B.; Mann, G. E.; Moore, K.; Roberts, L. J., 2nd; Ischiropoulos, H. Measuring Reactive Oxygen and Nitrogen Species with Fluorescent Probes: Challenges and Limitations. Free Radical Biol. Med. 2012, 52 (1), 1-6.

(54) Walsh, F. X.; Stevens, T. J.; Langlais, P. J.; Bird, E. D. Dopamine and Homovanillic Acid Concentrations in Striatal and Limbic Regions of Human Brain. Ann. Neurol. 1982, 12 (1), 52-5.

(55) Nutt, J. G.; Carter, J. H.; Sexton, G. J. The Dopamine Transporter: Importance in Parkinson's Disease. Ann. Neurol. 2004, 55 (6), 766-73.

(56) Jones, S. R.; Joseph, J. D.; Barak, L. S.; Caron, M. G.; Wightman, R. M. Dopamine Neuronal Transport Kinetics and Effects of Amphetamine. J. Neurochem. 1999, 73 (6), 2406-14.

(57) Nutt, J. G.; Wooten, G. F. Clinical Practice. Diagnosis and Initial Management of Parkinson's Disease. N. Engl. J. Med. 2005, 353 (10), 1021-7.

(58) Bolea, I.; Colivicchi, M. A.; Ballini, C.; Marco-Contelles, J.; Tipton, K. F.; Unzeta, M.; Della Corte, L. Neuroprotective Effects of the MAO-B Inhibitor, PF9601N, in an in Vivo Model of Excitotoxicity. CNS Neurosci. Ther. 2014, 20 (7), 641-50.

(59) Onzawa, Y.; Kimura, Y.; Uzuhashi, K.; Shirasuna, M.; Hirosawa, T.; Taogoshi, T.; Kihira, K. Effects of 3-O-Methyldopa, L-3,4-Dihydroxyphenylalanine Metabolite, on Locomotor Activity and Dopamine Turnover in Rats. Biol. Pharm. Bull. 2012, 35 (8), 1244-8.

(60) Werkman, T. R.; Glennon, J. C.; Wadman, W. J.; McCreary, A. C. Dopamine Receptor Pharmacology: Interactions with Serotonin Receptors and Significance for the Aetiology and Treatment of Schizophrenia. CNS Neurol. Disord.: Drug Targets 2006, 5 (1), 3-23.

(61) Eisenhofer, G.; Kopin, I. J.; Goldstein, D. S. Catecholamine Metabolism: a Contemporary View with Implications for Physiology and Medicine. Pharmacol. Rev. 2004, 56 (3), 331-49.

(62) Zhang, H.; Sulzer, D. Regulation of Striatal Dopamine Release by Presynaptic Auto- and Heteroreceptors. Basal ganglia 2012, 2 (1), 5-13.

(63) Gabriel, L. R.; Wu, S.; Kearney, P.; Bellve, K. D.; Standley, C.; Fogarty, K. E.; Melikian, H. E. Dopamine Transporter Endocytic Trafficking in Striatal Dopaminergic Neurons: Differential Dependence on Dynamin and the Actin Cytoskeleton. J. Neurosci. 2013, 33 (45), 17836-46.

(64) Hargraves, R.; Freed, W. J. Chronic Intrastriatal Dopamine Infusions in Rats with Unilateral Lesions of the Substantia Nigra. Life Sci. 1987, 40 (10), 959-66.

(65) Venna, N.; Sabin, T. D.; Ordia, J. I.; Mark, V. H. Treatment of Severe Parkinson's Disease by Intraventricular Injection of Dopamine. Appl. Neurophysiol. 1984, 47 (1-2), 62-4.

(66) Suarez-Garcia, S.; Arias-Ramos, N.; Frias, C.; Candiota, A. P.; Arus, C.; Lorenzo, J.; Ruiz-Molina, D.; Novio, F. Dual T1/ T2 Nanoscale Coordination Polymers as Novel Contrast Agents for MRI: A Preclinical Study for Brain Tumor. ACS Appl. Mater. Interfaces 2018, 10 (45), 38819-38832.
(67) Borges, M.; Laromaine, A.; Roig, A.; Suarez-Garcia, S.; Lorenzo, J.; Ruiz-Molina, D.; Novio, F. Dual T1/T2MRI Contrast Agent Based on Hybrid SPION@ Coordination Polymer Nanoparticles. RSC Adv. 2015, 5, 86779-86783.

(68) Guo, J.; Ping, Y.; Ejima, H.; Alt, K.; Meissner, M.; Richardson, J. J.; Yan, Y.; Peter, K.; von Elverfeldt, D.; Hagemeyer, C. E.; Caruso, F. Engineering Multifunctional Capsules Through the Assembly of Metal-Phenolic Networks. Angew. Chem., Int. Ed. 2014, 53 (22), $5546-51$.

(69) Wang, J.; de Keizer, A.; van Leeuwen, H. P.; Yan, Y.; Vergeldt, F.; van As, H.; Bomans, P. H.; Sommerdijk, N. A.; Cohen Stuart, M. A.; van der Gucht, J. Effect of $\mathrm{pH}$ on Complex Coacervate Core Micelles from $\mathrm{Fe}(\mathrm{III})$-Based Coordination Polymer. Langmuir 2011, 27 (24), 14776-82.

(70) Rodriguez, E.; Simoes, R. V.; Roig, A.; Molins, E.; Nedelko, N.; Slawska-Waniewska, A.; Aime, S.; Arus, C.; Cabanas, M. E.; Sanfeliu, C.; Cerdan, S.; Garcia-Martin, M. L. An Iron-Based T1 Contrast Agent Made of Iron-Phosphate Complexes: in Vitro and in Vivo Studies. MAGMA 2007, 20 (1), 27-37.

(71) Schwert, D. D.; Richardson, N.; Ji, G.; Raduchel, B.; Ebert, W.; Heffner, P. E.; Keck, R.; Davies, J. A. Synthesis of Two 3,5Disubstituted Sulfonamide Catechol Ligands and Evaluation of their Iron(III) Complexes for Use as MRI Contrast Agents. J. Med. Chem. 2005, 48 (23), 7482-5.

(72) Horcajada, P.; Chalati, T.; Serre, C.; Gillet, B.; Sebrie, C.; Baati, T.; Eubank, J. F.; Heurtaux, D.; Clayette, P.; Kreuz, C.; Chang, J. S.; Hwang, Y. K.; Marsaud, V.; Bories, P. N.; Cynober, L.; Gil, S.; Ferey, G.; Couvreur, P.; Gref, R. Porous Metal-Organic-Framework Nanoscale Carriers as a Potential Platform for Drug Delivery and Imaging. Nat. Mater. 2010, 9 (2), 172-8.

(73) Mustafi, D.; Peng, B.; Foxley, S.; Makinen, M. W.; Karczmar, G. S.; Zamora, M.; Ejnik, J.; Martin, H. New Vanadium-Based Magnetic Resonance Imaging Probes: Clinical Potential for Early Detection of Cancer. JBIC, J. Biol. Inorg. Chem. 2009, 14 (8), 118797.

(74) Marradi, M.; Alcantara, D.; de la Fuente, J. M.; Garcia-Martin, M. L.; Cerdan, S.; Penades, S. Paramagnetic Gd-Based Gold Glyconanoparticles as Probes for MRI: Tuning Relaxivities with Sugars. Chem. Commun. 2009, 26, 3922-4.

(75) Gheorghe, D. E.; Cui, L.; Karmonik, C.; Brazdeikis, A.; Penaloza, J. M.; Young, J. K.; Drezek, R. A.; Bikram, M. Gold-Silver Alloy Nanoshells: a New Candidate for Nanotherapeutics and Diagnostics. Nanoscale Res. Lett. 2011, 6, 554.

(76) Candiota, A. P.; Acosta, M.; Simoes, R. V.; Delgado-Goni, T.; Lope-Piedrafita, S.; Irure, A.; Marradi, M.; Bomati-Miguel, O.; Miguel-Sancho, N.; Abasolo, I.; Schwartz, S., Jr.; Santamaria, J.; Penades, S.; Arus, C. A New ex Vivo Method to Evaluate the Performance of Candidate MRI Contrast Agents: a Proof-of-Concept Study. J. Nanobiotechnol. 2014, 12, 12.

(77) Dhuria, S. V.; Hanson, L. R.; Frey, W. H., 2nd Intranasal Delivery to the Central Nervous System: Mechanisms and Experimental Considerations. J. Pharm. Sci. 2010, 99 (4), 1654-73.

(78) Dahlin, M.; Jansson, B.; Bjork, E. Levels of Dopamine in Blood and Brain Following Nasal Administration to Rats. Eur. J. Pharm. Sci. 2001, 14 (1), 75-80.

(79) Adarsh, N. N.; Ponnoth, T. M.; Lorenzo, J.; Novio, F.; GarciaPardo, J.; Ruiz-Molina, D. Pt (IV)-Based Nanoscale Coordination Polymers: Antitumor Activity, Cellular Uptake and Interactions with Nuclear DNA. Chem. Eng. J. 2018, 340, 94-102.

(80) Schneider, C. A.; Rasband, W. S.; Eliceiri, K. W. NIH Image to ImageJ: 25 Years of Image Analysis. Nat. Methods 2012, 9 (7), 6715.

(81) Kilts, C. D.; Breese, G. R.; Mailman, R. B. Simultaneous Quantification of Dopamine, 5-Hydroxytryptamine and Four Metabolically Related Compounds by Means of Reversed-Phase High-Performance Liquid Chromatography with Electrochemical Detection. J. Chromatogr., Biomed. Appl. 1981, 225 (2), 347-57. 
(82) Saldana-Ruiz, S.; Soler-Martin, C.; Llorens, J. Role of CYP2E1Mediated Metabolism in the Acute and Vvestibular Toxicities of Nineteen Nitriles in the Mouse. Toxicol. Lett. 2012, 208 (2), 125-32. (83) Gu, L.; Sailor, M. J.; Park, J. H. in Vivo Clearance and Toxicity of Monodisperse Iron Oxide Nanocrystals. ACS Nano 2012, 6, 4947-4954.

(84) Funk, F.; Ryle, P.; Canclini, C.; Neiser, S.; Geisser, P. The New Generation of Intravenous Iron: Chemistry, Pharmacology, and Toxicology of Ferric Carboxymaltose. Arzneim. Forsch. 2010, 60 (6a), 345-53.

(85) Bove, J.; Marin, C.; Bonastre, M.; Tolosa, E. Adenosine A2A Antagonism Reverses Levodopa-Induced Motor Alterations in Hemiparkinsonian Rats. Synapse 2002, 46 (4), 251-7.

(86) Paxinos, G.; Watson, C. The Rat Brain In Stereotaxic Coordinates; Academic Press: New York, 2013. 